\title{
A CONSTANT RANK THEOREM FOR HERMITIAN $k$-CONVEX SOLUTIONS OF COMPLEX LAPLACE EQUATIONS*
}

\author{
FEI HAN ${ }^{\dagger}$, XI-NAN MA ${ }^{\ddagger}$, AND DAMIN WU
}

\begin{abstract}
Using the strong maximum principle, we obtain a constant rank theorem for the Hermitian $k$-convex solutions of complex Laplace equation.
\end{abstract}

Key words. Hermitian $k$-convex, constant rank, complex Laplace equation, maximum principle.

AMS subject classifications. 35J60, 58G11, 32Q15

1. Introduction. In some geometry and analysis problems, it is always important to get the existence of convex solutions for partial differential equations. Up to now, we have in general two methods to produce convex solutions for elliptic partial differential equations. They are the macroscopic and microscopic convexity principle.

The macroscopic convexity principle was developed by Korevaar [19, 20], Kennington [17], Kawohl [16], and by Alvarez-Lasry-Lions [1] for generally nonlinear partial differential equations.

The microscopic convexity principle concentrates on the constant rank theorem for convex solutions of partial differential equations. Caffarelli-Friedman [4] proved a constant rank theorem for convex solutions of quasilinear elliptic equations in $\mathbb{R}^{2}$; a similar result was also obtained by Yau [28] (in fact, Yau have known the idea much earlier, according to A. Treibergs, and also Korevaar [21, p. 117].). The method of Caffarelli-Friedman-Yau was generalized to $\mathbb{R}^{n}$ by Korevaar-Lewis [22] shortly after. It is a powerful tool in producing convex solutions of partial differential equations via the continuity methods. The microscopic convexity principle has been generalized to a variety of fully nonlinear differential equations involving the second fundamental forms of hypersurfaces (see, for instance, [5, 11, 12].). Recently, Bian-Guan [2] obtained the microscopic convexity principle for the most generally fully nonlinear differential equations and gave much interesting geometry applications. It is interesting that the structure condition found by Bian-Guan [2] are almost the same as the structure condition that found by Alvarez-Lasry and Lions in [1].

In differential geometry, there are much interesting on the $k$-convexity of the solutions of the geometric partial differential equations. Here the $k$-convexity means that the sum of any $k$ eigenvalues of the Hessian matrix of the solution is nonnegative. Similarly, we can formulate the notions of $k$-convexity for the curvature operator and second fundamental forms of hypersurfaces. There has been a large amount of literature in differential geometry on this subject. For example, Sha $[26,27]$ and $\mathrm{H}$. Wu [30] introduced the $k$-positivity of the sectional curvature of Riemannian manifolds and studied the topology for these manifolds. Chen [7] and Böhm-Wilking [3] studied

\footnotetext{
*Received November 25, 2008; accepted for publication April 15, 2009.

$\dagger$ Department of Mathematics, University of Science and Technology of China, Hefei, 230026, Anhui Province, China; and College of Mathematics, Physics and Information, Xinjiang Normal University, Urumqi, 830054, Xinjiang Uygur Autonomous Region, China (klhqf@mail.ustc.edu.cn).

${ }^{\ddagger}$ Department of Mathematics, University of Science and Technology of China, Hefei, 230026, Anhui Province, China (xinan@ustc.edu.cn). Research of the second author was supported by NSFC No. 10671186 and "Hundreds Peoples Program in Chinese Academy of Sciences".

$\S$ Department of Mathematics, The Ohio State University, 1179 University Drive, Newark, OH 43055, USA (dwu@math.ohio-state.edu).
} 
the classification of compact Riemannian manifolds with 2-positive curvature operator via Ricci flow. Moore-Schulte [25] and Fraser [9, 10] obtained some Morse index estimates for the minimal disk with free boundary on the $k$-convex surfaces and then have some interesting consequences on the topology of $k$-convex domain in $\mathbb{R}^{n}$. More recently, Huisken-Sinestrari [15] classified the compact 2-convex hypersurfaces in $\mathbb{R}^{n}$ using the technique of mean curvature flow. Let us also call attention to the work by Tsui and Wang [29], in which the 2-positivity of some parallel 2-tensor on manifolds plays an important role on the long time existence and convergence of mean curvature flows.

In the study of elliptic equations, Harvey-Lawson [14] introduced some generally convexity on the solutions of the nonlinear elliptic Dirichlet problem. The $k$-convexity is a special case.

From the above geometry and analysis reasons, it is naturally to study the general microscopic and macroscopic convexity principle in the context of the $k$-convex solutions of partial differential equations. In Han-Ma-Wu [13] we establish a constant rank theorem of the $k$-convex hypersurfaces on the prescribed mean curvature equation in $\mathbb{R}^{n+1}$, and then as an application, we obtain an existence theorem for $k$-convex hypersurfaces with prescribed mean curvature.

For the potential application and independent interesting in complex analysis and complex geometry, in this paper we establish the complex counterpart of the constant rank theorem on the Hermitian $k$-convex solutions for complex Laplace equation. The constant rank theorem on the Hermitian 1-convex (i.e., plurisubharmonic) solutions for some elliptic partial differential equations in complex variables had been considered by Q. Li in [23]. We will see from below that the ranks of $k-$ convex $(k \geq 2)$ solutions enjoy certain interesting jumping phenomenon. This would distinguish our treatment from those in the literature. The Hermitian $k$-convexity $(k \geq 2)$ has naturally appeared, and played an important role, in the several complex variables and complex geometry, such as the vanishing theorem (see, for example, Kobayashi-Wu [18], and Ma-Marinescu [24]), and the Cauchy-Riemann geometry (see, for example, Chirka [8]). We shall report the applications of our result in a later occasion.

We consider the following equation

$$
\Delta u=\sum_{\alpha=1}^{n} \frac{\partial^{2} u}{\partial z^{\alpha} \partial \bar{z}^{\alpha}}=f(z)
$$

in a domain $\Omega$ of $\mathbb{C}^{n}$. Here we assume that

$$
f \in C^{2, \lambda}(\Omega), \quad f>0, \quad \text { for some } 0<\lambda<1 .
$$

We are interested in the solution $u$ of Hermitian $k$-convex. Namely, the sum of any $k$ eigenvalues of the Hermitian matrix $\left(u_{\alpha \bar{\beta}}\right)_{1 \leq \alpha, \beta \leq n}$ is nonnegative. We would like to know, under what condition would the Hermitian $k$-convex solution $u$ has certain constant rank in $\Omega$. Throughout this note, $k$ denotes an integer in $\{1, \ldots, n-1\}$ (there is nothing to prove when $k=n$, since $f>0$ by our assumption.).

It is convenient to work with the following $w$-matrix, whose eigenvalues are the sums of the $k$ eigenvalues of $\left(u_{\alpha \bar{\beta}}\right)$. First, note that $\left(u_{\alpha \bar{\beta}}\right)$ induces an operator $P$ on $\mathbb{C}^{n}$ by

$$
P\left(e_{\alpha}\right)=\sum_{\gamma=1}^{n} u_{\gamma \bar{\alpha}} e_{\gamma}, \quad \text { for all } 1 \leq \alpha \leq n
$$


where $\left\{e_{1}, \ldots, e_{n}\right\}$ is the standard basis of $\mathbb{C}^{n}$, as a complex vector space. As in Caffarelli-Nirenberg-Spruck [6], we further extend $P$ to acting on the complex vector space $\wedge^{k} \mathbb{C}^{n}$ by

$$
P\left(e_{\alpha_{1}} \wedge \cdots \wedge e_{\alpha_{k}}\right)=\sum_{i=1}^{k} e_{\alpha_{1}} \wedge \cdots \wedge P\left(e_{\alpha_{i}}\right) \wedge \cdots \wedge e_{\alpha_{k}} .
$$

Here $\left\{e_{\alpha_{1}} \wedge \cdots \wedge e_{\alpha_{k}} \mid 1 \leq \alpha_{1}<\cdots<\alpha_{k} \leq n\right\}$ is the standard basis for $\wedge^{k} \mathbb{C}^{n}$. Let $\left(w_{i j}\right)$ be the matrix of $P$ under this standard basis, where the indices $i$ and $j$ range from 1 to $\left(\begin{array}{l}n \\ k\end{array}\right)$. It is, however, more helpful to write the indices of $w$ in terms of the base indices. Namely, we denote by

$$
w_{\alpha_{1} \ldots \alpha_{k}, \beta_{1} \ldots \beta_{k}}=\left\langle P\left(e_{\alpha_{1}} \wedge \cdots \wedge e_{\alpha_{k}}\right), e_{\beta_{1}} \wedge \cdots \wedge e_{\beta_{k}}\right\rangle .
$$

Here $\langle\cdot, \cdot\rangle$ is the inner product on $\wedge^{k} \mathbb{C}^{n}$ induced from the standard one on $\mathbb{C}^{n}$ so that

$$
\left\langle a e_{\alpha_{1}} \wedge \cdots \wedge e_{\alpha_{k}}, b e_{\beta_{1}} \wedge \cdots \wedge e_{\beta_{k}}\right\rangle=\delta\left(\begin{array}{c}
\alpha_{1}, \ldots, \alpha_{k} \\
\beta_{1}, \ldots, \beta_{k}
\end{array}\right) a \bar{b},
$$

in which $a, b \in \mathbb{C}$. We denote by $\delta\left(\begin{array}{l}a_{1}, \ldots, a_{k} \\ b_{1}, \ldots, b_{k}\end{array}\right)$ the generalized Kronecker symbol; it is equal to 1 (respectively, -1 ) if $a_{1}, \ldots, a_{k}$ are distinct and $\left(b_{1}, \ldots, b_{k}\right)$ is an even (respectively, odd) permutation of $\left(a_{1}, \ldots, a_{k}\right)$; otherwise it is equal to zero. Then,

$$
w_{\alpha_{1} \ldots \alpha_{k}, \beta_{1} \ldots \beta_{k}}=\sum_{i=1}^{k} \sum_{\gamma=1}^{n} u_{\gamma \bar{\alpha}_{i}} \delta\left(\begin{array}{c}
\alpha_{1}, \ldots, \alpha_{i-1}, \gamma, \alpha_{i+1}, \ldots, \alpha_{k} \\
\beta_{1}, \ldots, \beta_{i-1}, \beta_{i}, \beta_{i+1} \ldots, \beta_{k}
\end{array}\right) .
$$

In particular,

$$
w_{\alpha_{1} \ldots \alpha_{k}, \alpha_{1} \ldots \alpha_{k}}=\sum_{i=1}^{k} u_{\alpha_{i} \bar{\alpha}_{i}}
$$

and

$$
w_{\alpha_{1} \ldots \alpha_{k}, \beta_{1} \ldots \beta_{k}}=0
$$

if the index sets $\left\{\alpha_{1}, \ldots, \alpha_{k}\right\}$ and $\left\{\beta_{1}, \ldots, \beta_{k}\right\}$ are differed by two elements. It follows that the $w$-matrix is Hermitian, i.e.,

$$
w_{\alpha_{1} \ldots \alpha_{k}, \beta_{1} \ldots \beta_{k}}=\bar{w}_{\beta_{1} \ldots \beta_{k}, \alpha_{1} \ldots \alpha_{k}} .
$$

Moreover, if we normalize the $u$-matrix at one point so that

$$
u_{\alpha \bar{\beta}}=\delta_{\alpha \beta} u_{\alpha \bar{\alpha}},
$$

then the $w$-matrix is also diagonalized:

$$
w_{\alpha_{1} \ldots \alpha_{k}, \beta_{1} \ldots \beta_{k}}=\left(\prod_{i=1}^{k} \delta_{\alpha_{i} \beta_{i}}\right) w_{\alpha_{1} \ldots \alpha_{k}, \alpha_{1} \ldots \alpha_{k}}=\prod_{i=1}^{k} \delta_{\alpha_{i} \beta_{i}} \sum_{i=1}^{k} u_{\alpha_{i} \bar{\alpha}_{i}} .
$$

As an example, when $k=2$, we have

$$
w_{\alpha \beta, \eta \zeta}=\left\langle P\left(e_{\alpha} \wedge e_{\beta}\right), e_{\eta} \wedge e_{\zeta}\right\rangle .
$$


Then

$$
\begin{aligned}
w_{\alpha \beta, \eta \zeta} & =\delta_{\beta \zeta} u_{\eta \bar{\alpha}}-\delta_{\beta \eta} u_{\zeta \bar{\alpha}}+\delta_{\alpha \eta} u_{\zeta \bar{\beta}}-\delta_{\alpha \zeta} u_{\eta \bar{\beta}} \\
& =\bar{w}_{\eta \zeta, \alpha \beta} .
\end{aligned}
$$

In particular, the diagonal elements are given by

$$
w_{\alpha \beta, \alpha \beta}=u_{\alpha \bar{\alpha}}+u_{\beta \bar{\beta}} .
$$

Throughout this note, we denote by

$$
N=\operatorname{dim}_{\mathbb{C}} \wedge^{k} \mathbb{C}^{n}=\left(\begin{array}{l}
n \\
k
\end{array}\right)=\frac{n !}{k !(n-k) !} .
$$

Unless otherwise indicated, the Greek indices $\alpha, \beta, \gamma, \ldots$, are in $\{1, \ldots, n\}$, while English indices $i, j, l, \ldots$, lie in $\{1, \ldots, N\}$. We may interchangeably use $\left(w_{i j}\right)$ and $\left(w_{\alpha_{1} \ldots \alpha_{k}, \beta_{1} \ldots \beta_{k}}\right)$ to indicate the $w$-matrix. That is, we may use a single index, such as $i$, to represent a multi-index $\left(\alpha_{1}, \ldots, \alpha_{k}\right)$. The correspondence is given by the oneto-one map from $\mathcal{S}=\{1, \ldots, N\}$ to $\mathcal{M}=\left\{\left(\alpha_{1}, \ldots, \alpha_{k}\right) \mid 1 \leq \alpha_{1}<\cdots<\alpha_{k} \leq n\right\}$, preserving the order of the elements. Here the order in $\mathcal{S}$ is as usual, while the order in $\mathcal{M}$ is defined to be that

$$
\left(\alpha_{1}, \ldots, \alpha_{k}\right) \prec\left(\beta_{1}, \ldots, \beta_{k}\right)
$$

if there is a number $q \in\{1, \ldots, n\}$ such that $\alpha_{q}<\beta_{q}$ and $\alpha_{j}=\beta_{j}$ for all $j=$ $1, \ldots, q-1$. For example, when $n=3$ and $k=2$, the correspondence are identified as

$$
1=(1,2), \quad 2=(1,3), \quad 3=(2,3) ;
$$

and hence,

$$
w_{11}=w_{12,12}, \quad w_{12}=w_{12,13}, \quad w_{13}=w_{12,23}, \quad \cdots .
$$

However, a precise formula of the correspondence is not needed in the follows.

For a finite set $A$, we denote by $\# A$ the total number of elements in $A$. For derivatives of a function $h$, we denote by

$$
h_{\alpha}=\frac{\partial h}{\partial z^{\alpha}}, \quad h_{\bar{\alpha}}=\frac{\partial h}{\partial \bar{z}^{\alpha}}, \quad h_{\alpha \bar{\beta}}=\frac{\partial^{2} h}{\partial z^{\alpha} \bar{z}^{\beta}}, \quad \cdots .
$$

A Hermitian matrix $\left(H_{\alpha \bar{\beta}}\right)$ is called $k$-concave, if the sum of any $k$ eigenvalues of $\left(H_{\alpha \bar{\beta}}\right)$ is nonpositive, equivalently, if $\lambda_{1}+\cdots+\lambda_{k} \leq 0$, where $\lambda_{1} \geq \lambda_{2} \geq \cdots \geq \lambda_{n}$ are the eigenvalues of $\left(H_{\alpha \bar{\beta}}\right)$. In particular, a 1-concave Hermitian matrix is nonpositive definite. A $C^{2}$-function $v$ is called Hermitian $k$-concave in $\Omega$ if $\left(v_{\alpha \bar{\beta}}\right)$ is $k$-concave in $\Omega$. Here is our main result:

THEOREM 1.1. Let $u$ and $f$ satisfy (1.1), (1.2). Suppose that the $w$-matrix is positive semidefinite on $\Omega$. Then the $w$-matrix has constant rank in $\Omega$, if the Hermitian matrix

$$
\left(k f f_{\alpha \bar{\beta}}-f_{\alpha} f_{\bar{\beta}}\right)_{1 \leq \alpha, \beta \leq n}
$$

is $k$-concave in $\Omega$. Equivalently, the $w$-matrix has constant rank if $f^{1-1 / k}$ is Hermitian $k$-concave for $k \geq 2$, and $-\log f$ is plurisubharmonic when $k=1$. 
REMARK 1.2. In the above theorem, the $k=1$ case was obtained by $Q$. Li in [23]. For $k \geq 2$, there are restrictions on the possible ranks of $w$ (these restrictions are explicitly given in the proof.). Consider $k=2$ for instance: The possible ranks of $w$ must lie in

$$
\{N-\kappa(\kappa+1) / 2 \mid \kappa=1, \ldots, n-2\} \cup\{N-n, \ldots, N\} .
$$

Furthermore, if $n=6$ for example, then the possible ranks are in the set

$$
\{5\} \cup\{r \in \mathbb{Z} \mid 9 \leq r \leq 15\} .
$$

Our proof would follow the standard approach in the literature (see KorevaarLewis [22] and Guan-Ma [12], for example). Assume that $\left(w_{i j}\right)$ has rank $r<N$ at a point. Let $\sigma_{r+1}$ be the sum of all $r+1$ by $r+1$ principal minors of $\left(w_{i j}\right)$. The main step is to derive the estimate

$$
\Delta \sigma_{r+1} \leq c_{1}\left|\nabla \sigma_{r+1}\right|+c_{2} \sigma_{r+1}
$$

where the constants $c_{1}$ and $c_{2}$ are under control up to the $\|u\|_{C^{4}}$. Then the desired result would follow from the strong maximum principle.

The major difference between the $w$-matrix, and the $u$-matrix (which was treated in literature, such as Korevaar-Lewis [22]), is that the indices of $w$ cannot commute, such as $w_{i j ; \alpha} \neq w_{i \alpha ; j}$. Thus, one has to convert $w$ to $u$; in particular, one has to find their correspondences of the "good" or "bad" sets of indices (see the next section for the definitions). Given a general rank, it is not easy to determine the "good" or "bad" indices for $w$, because one can never expect to order the diagonal elements of $w$ completely in terms of the indices, even though we can do that for $u$, say (3.1). We do have some partial orders for $w$, however.

The key observation here is that the "bad" indices can only appear in certain explicit regular cases, because of the partial orders of $w_{i i}$. In the proof we divide the "bad" sets into two cases. Geometrically, one is the solid "cone" while the other consists of certain boundaries of the "cone" in the index space. In particular, this imposes some restrictions on the possible ranks on the $w$-matrix. As a consequence, we do not have to derive for each $\sigma_{r+1}$ the hard estimates. More importantly, the explicit "bad" sets of indices make it much easier to transfer from $w$ to $u$. This greatly simplifies the calculations.

For instance, for $\wedge^{2} \mathbb{C}^{6}$, the possible "bad" sets are given as follows:

$$
B_{1, \mu}=\{(\alpha, \beta) \mid \mu \leq \alpha<\beta \leq 6\}, \quad \text { for } \mu=2,3,4,
$$

and

$$
B_{2, \nu}=\{(\nu, 6),(\nu+1,6), \ldots,(5,6)\}, \quad \text { for } \nu=1,2, \ldots, 5 .
$$

In particular, this shows that the rank of $w$-matrix at any point can never be $1,2,3$, $4,6,7,8$.

Comparing with [13] in the real case, the Hermitian $k$-convexity is weaker than the $k$-convexity of the full Hessian. In the complex case one has to distinguish the indices of $u$ with bars and without bars, especially in the third derivatives. Roughly speaking, we would drop the terms as $\left|u_{\alpha \alpha \bar{\beta}}\right|^{2}$ while keep the terms $\left|u_{\alpha \bar{\alpha} \bar{\beta}}\right|^{2}$ in the derivation. This is one of the reasons why the powers in the concavity of $f$ are shifted by $1 / k$ in the main results. 
Let us finally remark that, the Hermitian $k$-concavity of $f^{1-1 / k}, k \geq 2$, is only needed in the second case to establish (1.4), while a weaker assumption, such as the Hermitian $k$-concavity of $\log f$, is enough for the first case of the "bad" sets (see Lemma 3.4 and Lemma 4.6 below).

In Section 2 certain estimates of $w$ are established for later use. To make it easier for the reader to comprehend the idea, we first settle Theorem 1.1 for $k=2$ in Section 3. The general case is proved in the last section.

Acknowledgment. Part of the work was done while the third author was visiting the University of Science and Technology of China in July 2008, he would like to thank their warm hospitality. The second author would also like to thank Professor P. Guan for his encouragement and support.

2. Estimates. In this section we shall reduce the proof of Theorem 1.1 to establishing certain Laplacian estimates. Throughout this section, we denote the Hermitian $w$-matrix by $\left(w_{i j}\right)$. The English letters, $i, j, l, \ldots$, range from 1 to $N$, and the Greek letters, $\alpha, \beta, \gamma, \ldots$, range from 1 to $n$, unless otherwise indicated.

By (1.1) we have

$$
\sum_{i=1}^{N} w_{i i}=\left(\begin{array}{l}
n-1 \\
k-1
\end{array}\right) f, \quad \text { in } \Omega .
$$

Since $f>0$, the rank of $\left(w_{i j}\right)$ is at least 1 everywhere in $\Omega$. Suppose that $\left(w_{i j}\right)$ attains its minimal rank $r<N$ at some point $x_{0}$ in $\Omega$. Let $\phi=\sigma_{r+1}$, the sum of all $r+1$ by $r+1$ principal minors of $\left(w_{i j}\right)$. Our goal is to show that $\phi$ vanishes identically in $\Omega$.

We first to show that $\phi \equiv 0$ in a neighborhood of $x_{0}$. For two real-valued functions $h$ and $g$ defined in a neighborhood of $x_{0}$, we write $h \lesssim g$, if

$$
(h-g)(x) \leq\left(c_{1}|\nabla \phi|+c_{2} \phi\right)(x)
$$

for any $x$ in this neighborhood, where $c_{1}$ and $c_{2}$ are two positive constants independent of $x$. We write $h \sim g$ if $h \lesssim g$ and $g \lesssim h$. We also denote by $h(x) \lesssim g(x)$ the pointwise estimate (2.1), and similarly for $h(x) \sim g(x)$. For complex-valued functions $g$ and $h$, we write $g \sim h$ if $|g-h| \lesssim 0$. In the following, all calculations are carried out at one point using the relation $\underset{\lesssim}{\lesssim}$ with the understanding that the constants in (2.1) are under control.

Note that we only have to show that

$$
\Delta \phi=\sum_{\alpha=1}^{n} \phi_{\alpha \bar{\alpha}} \lesssim 0
$$

Indeed, since $\phi \geq 0$ and $\phi\left(x_{0}\right)=0$, applying the strong maximum principle to (2.2) yields that $\phi \equiv 0$ in a neighborhood of $x_{0}$.

Thus, by assuming (2.2) we conclude that $\phi \equiv 0$ locally. Recall that $r$ is the minimal rank of $\left(w_{i j}\right)$. Hence, the subset

$$
\Omega_{r}=\left\{y \in \Omega \mid \operatorname{rank}\left(w_{i j}\right)(y)=r\right\}
$$

is open. On the other hand, the continuity of $\phi$ implies that

$$
\operatorname{rank}\left(w_{i j}\right)\left(x_{1}\right) \leq r
$$


for any $x_{1} \in \Omega \cap \partial \Omega_{r}$. But $r$ is the minimal rank; this forces that

$$
\operatorname{rank}\left(w_{i j}\right)\left(x_{1}\right)=r
$$

Hence, $\Omega_{r}$ is also closed in $\Omega$. Therefore, $\Omega_{r}=\Omega$, in view of the connectivity of $\Omega$. Therefore, the proof of Theorem 1.1 is reduced to establishing (2.2).

To verify (2.2), let us introduce the "good" and "bad" sets of indices, and then derive certain estimates related to these sets. Recall that $\left(w_{i j}\right)$ has rank $r$ at $x_{0}$. We can pick a constant $c>0$ so that the $r$ nonzero eigenvalues of $\left(w_{i j}\right)\left(x_{0}\right)$ are bounded below by $2 c$. Then, by continuity $\left(w_{i j}\right)(x)$ has exactly $r$ eigenvalues $\geq c$, for any point $x$ near $x_{0}$. Fix such an $x$. We choose a coordinate system so that $\left(u_{\alpha \bar{\beta}}\right)$ is diagonalized at $x$; then so is $\left(w_{i j}\right)$. We denote by

$$
G=\left\{i \mid w_{i i}(x) \geq c, 1 \leq i \leq N\right\}
$$

and

$$
B=\{1, \ldots, N\} \backslash G .
$$

In other words, $G$ consists of the indices of the eigenvalues of $w$-matrix which is no less than $c$, and $B$ is the complement of $G$ in the total index set. Then, $\# G=r$ and $\# B=N-r$. We call $G$ and $B$, respectively, the "good" and "bad" sets of indices. We remark that, if one works with the multiple indices of $w$, i.e., $\left(w_{\alpha_{1} \ldots \alpha_{k}, \beta_{1} \ldots \beta_{k}}\right)$, , then

$$
G=\left\{\left(\alpha_{1}, \ldots, \alpha_{k}\right) \mid w_{\alpha_{1} \ldots \alpha_{k}, \alpha_{1} \ldots \alpha_{k}}(x) \geq c, 1 \leq \alpha_{1}<\cdots<\alpha_{k} \leq n\right\}
$$

and

$$
B=\left\{\left(\alpha_{1}, \ldots, \alpha_{k}\right) \mid 1 \leq \alpha_{1}<\cdots<\alpha_{k} \leq n\right\} \backslash G .
$$

Let us start with some simple observations, which will be used in the later reduction.

Proposition 2.1. Let $G$ and $B$ be defined as in (2.3) and (2.4), respectively. Let $i$ be an element in $G$. Then $w_{i i}$ is not $\sim 0$. If for some function $h$ defined in a neighborhood of $x_{0}$ and $x$ such that $w_{i i} h \sim 0$, then $h \sim 0$.

Proof. By continuity, there exists a neighborhood $V$ of $x_{0}$ and $x$ such that

$$
w_{j j}(y) \geq c / 2, \quad \text { for all } y \in V \text { and } j \in G .
$$

Here the constant $c>0$ is given in (2.3). Now suppose that $w_{i i} \sim 0$ for some $i \in G$. This implies that

$$
w_{i i} \leq\left(c_{1}|\nabla \phi|+c_{2} \phi\right)
$$

in a neighborhood of $x_{0}$ and $x$, where $c_{1}$ and $c_{2}$ are constants independent of $x_{0}$ and $x$. Then, at the point $x_{0}$ we get a contradiction

$$
c / 2 \leq w_{i i}\left(x_{0}\right) \leq\left(c_{1}\left|\nabla \phi\left(x_{0}\right)\right|+c_{2} \phi\left(x_{0}\right)\right)=0,
$$

where the last equality holds because $x_{0}$ is a minimum point of $\phi$. This proves the first statement. The second statement follows immediately from (2.5) and the definition of $\sim$. 
As in [22] the following proposition is standard.

Proposition 2.2. Let $G$ and $B$ be defined as in (2.3) and (2.4), respectively. Then,

$$
\begin{aligned}
w_{j j} & \sim 0, \quad \text { for all } j \in B, \\
\sum_{j \in B}\left(w_{j j}\right)_{\alpha} & \sim 0, \quad \sum_{j \in B}\left(w_{j j}\right)_{\bar{\alpha}} \sim 0,
\end{aligned}
$$

and

$$
\phi_{\alpha \bar{\alpha}} \lesssim \prod_{l \in G} w_{l l}\left(\sum_{j \in B}\left(w_{j j}\right)_{\alpha \bar{\alpha}}-\sum_{i \in G} \sum_{j \in B} \frac{\left|\left(w_{i j}\right)_{\alpha}\right|^{2}+\left|\left(w_{i j}\right)_{\bar{\alpha}}\right|^{2}}{w_{i i}}\right)
$$

for each $\alpha=1, \ldots, n$.

Proof. Note that $w_{i i} \geq 0$ for all $i=1, \ldots, N$. For each $j \in B$, we have

$$
0 \sim \phi \sim \prod_{i \in G} w_{i i} \cdot \sum_{l \in B} w_{l l} \geq w_{j j} \prod_{i \in G} w_{i i} \geq 0 .
$$

This implies that

$$
w_{j j} \prod_{i \in G} w_{i i} \sim 0
$$

Thus, we have $w_{j j} \sim 0$, by the second statement of Proposition 2.1. Next, for each $\alpha=1, \ldots, n$,

$$
0 \sim \phi_{\alpha} \sim \prod_{i \in G} w_{i i} \cdot \sum_{j \in B}\left(w_{j j}\right)_{\alpha} .
$$

Then, by the second statement of Proposition 2.1, we have for each $\alpha$

$$
\sum_{j \in B}\left(w_{j j}\right)_{\alpha} \sim 0, \quad \text { and } \quad \sum_{j \in B}\left(w_{j j}\right)_{\bar{\alpha}} \sim 0 .
$$

These prove (2.7).

Let us now compute $\phi_{\alpha \bar{\alpha}}$. By (2.3) and (2.4) we obtain

$$
\begin{aligned}
& \phi_{\alpha \bar{\alpha}}(x) \sim \prod_{i \in G} w_{i i}\left\{\sum_{j \in B}\left(w_{j j}\right)_{\alpha \bar{\alpha}}\right. \\
& +\frac{1}{2} \sum_{l \in G} \frac{1}{w_{l l}} \cdot \sum_{p, q \in B, p \neq q}\left\{2 \operatorname{Re}\left[\left(w_{p p}\right)_{\alpha}\left(w_{q q}\right)_{\bar{\alpha}}\right]-\left|\left(w_{p q}\right)_{\alpha}\right|^{2}-\left|\left(w_{p q}\right)_{\bar{\alpha}}\right|^{2}\right\} \\
& \left.+\sum_{l \in G} \sum_{p \in B} \frac{2 \operatorname{Re}\left[\left(w_{l l}\right)_{\alpha}\left(w_{p p}\right)_{\bar{\alpha}}\right]-\left|\left(w_{l p}\right)_{\alpha}\right|^{2}-\left|\left(w_{l p}\right)_{\bar{\alpha}}\right|^{2}}{w_{l l}}\right\} .
\end{aligned}
$$

By (2.7) we have

$$
\sum_{q \in B, q \neq p}\left(w_{q q}\right)_{\bar{\alpha}} \sim-\left(w_{p p}\right)_{\bar{\alpha}} .
$$


This implies that

$$
\sum_{p, q \in B, p \neq q} 2 \operatorname{Re}\left[\left(w_{p p}\right)_{\alpha}\left(w_{q q}\right)_{\bar{\alpha}}\right] \sim-2 \sum_{p \in B}\left|\left(w_{p p}\right)_{\alpha}\right|^{2} \leq 0 .
$$

Substituting this back to $\phi_{\alpha \bar{\alpha}}$ and applying (2.7) to the third term yield the desired result.

For later uses, let us rewrite the above results in terms of the multiple indices of $w$. That is,

$$
\sum_{\left(\beta_{1}, \ldots, \beta_{k}\right) \in B} \begin{aligned}
w_{\beta_{1} \ldots \beta_{k}, \beta_{1} \ldots \beta_{k}} & \sim 0, \quad \text { for all }\left(\beta_{1}, \ldots, \beta_{k}\right) \in B \\
\left(w_{\beta_{1} \ldots \beta_{k}, \beta_{1} \ldots \beta_{k}}\right)_{\alpha} & \sim \sum_{\left(\beta_{1}, \ldots, \beta_{k}\right) \in B}\left(w_{\beta_{1} \ldots \beta_{k}, \beta_{1} \ldots \beta_{k}}\right)_{\bar{\alpha}} \sim 0
\end{aligned}
$$

and

$$
\begin{aligned}
\phi_{\alpha \bar{\alpha}} & \lesssim Q\left(\sum_{\left(\beta_{1}, \ldots, \beta_{k}\right) \in B}\left(w_{\beta_{1} \ldots \beta_{k}, \beta_{1} \ldots \beta_{k}}\right)_{\alpha \bar{\alpha}}\right. \\
& \left.-\sum_{\left(\gamma_{1}, \ldots, \gamma_{k}\right) \in G} \sum_{\left(\beta_{1}, \ldots, \beta_{k}\right) \in B} \frac{\left|\left(w_{\beta_{1} \ldots \beta_{k}, \gamma_{1} \ldots \gamma_{k}}\right)_{\alpha}\right|^{2}+\left|\left(w_{\beta_{1} \ldots \beta_{k}, \gamma_{1} \ldots \gamma_{k}}\right) \bar{\alpha}\right|^{2}}{w_{\gamma_{1} \ldots \gamma_{k}, \gamma_{1} \ldots \gamma_{k}}}\right)
\end{aligned}
$$

for each $\alpha=1, \ldots, n$. Here and after, we denote by

$$
Q=\prod_{i \in G} w_{i i}=\prod_{\left(\gamma_{1}, \ldots, \gamma_{k}\right) \in G} w_{\gamma_{1} \ldots \gamma_{k}, \gamma_{1} \ldots \gamma_{k}} .
$$

3. Two-convex case. In this section, we shall prove Theorem 1.1 for the case $k=2$. Namely,

Theorem 3.1. Let $u$ and $f$ satisfy (1.1), (1.2). Suppose that the $\left(w_{\beta \gamma, \eta \zeta}\right) \geq 0$ on $\Omega$. Then $\left(w_{\beta \gamma, \eta \zeta}\right)$ has constant rank in $\Omega$, if $f^{1 / 2}$ is Hermitian 2 -concave in $\Omega$.

From the proof, we can say more about the (constant) ranks of $\left(w_{\beta \gamma, \eta \zeta}\right)$. Indeed, instead of being an arbitrary integer from 1 to $N$, the rank of $\left(w_{\beta \gamma, \eta \zeta}\right)$ must lie in

$$
\{N-n, \ldots, N\} \cup\{N-\kappa(\kappa+1) / 2 \mid \kappa=1, \ldots, n-2\} .
$$

Hereafter, we always work on the $w$-matrix with the multiple indices. In view of the previous section, we only have to derive (2.2) at the point $x$. We have chosen a local coordinate system so that

$$
u_{\alpha \bar{\beta}}(x)=\delta_{\alpha \beta} u_{\alpha \bar{\alpha}}(x)
$$

then $w_{\alpha \beta, \eta \zeta}$ at $x$ is also diagonalized to be

$$
w_{\alpha \beta, \eta \zeta}=\delta_{\alpha \eta} \delta_{\beta \zeta}\left(u_{\alpha \bar{\alpha}}+u_{\beta \bar{\beta}}\right)=\delta_{\alpha \eta} \delta_{\beta \zeta} w_{\alpha \beta, \alpha \beta} .
$$

Furthermore, we can arrange for $u$ that

$$
u_{1 \overline{1}}(x) \geq u_{2 \overline{2}}(x) \geq \cdots \geq u_{n \bar{n}}(x) .
$$


As before, we define the good and bad sets of indices to be, respectively,

$$
G=\left\{(\alpha, \beta) \mid w_{\alpha \beta, \alpha \beta}(x) \geq c, 1 \leq \alpha<\beta \leq n\right\},
$$

and

$$
B=\{(\alpha, \beta) \mid 1 \leq \alpha<\beta \leq n\} \backslash G .
$$

We claim that any bad set must be one of the following two cases:

(i) There exists some positive integer $\mu \leq n-2$ such that

$$
B=\{(\alpha, \beta) \mid \mu \leq \alpha<\beta \leq n\} .
$$

(ii) The bad set $B$ is a subset of $\{(\alpha, n) \mid \alpha=1, \ldots, n-1\}$.

Assuming this claim, then we only need to verify (2.2) for the above two cases. Furthermore, this claim imposes some constraints on the possible ranks of $w$ at any point. In particular, by this claim we know that

$$
\# B \in\{1, \ldots, n\} \cup\{\kappa(\kappa+1) / 2 \mid \kappa=1, \ldots, n-2\} .
$$

Therefore, the rank of $w$-matrix can only be in the following set:

$$
\{N-n, \ldots, N\} \cup\{N-\kappa(\kappa+1) / 2 \mid \kappa=1, \ldots, n-2\} .
$$

Before proving this claim, let us first derive from Proposition 2.2 certain simple properties regarding these sets. For $k=2$, the results of Proposition 2.2 can be reformulated as follows:

$$
\begin{aligned}
u_{\eta \bar{\eta}}+u_{\zeta \bar{\zeta}} & =w_{\eta \zeta, \eta \zeta} \sim 0, \quad \text { for all }(\eta, \zeta) \in B \\
\sum_{(\eta, \zeta) \in B}\left(u_{\eta \bar{\eta} \alpha}+u_{\zeta \bar{\zeta} \alpha}\right) & =\sum_{(\eta, \zeta) \in B}\left(w_{\eta \zeta, \eta \zeta}\right)_{\alpha} \sim 0
\end{aligned}
$$

and

$$
\phi_{\alpha \bar{\alpha}} Q^{-1} \lesssim \sum_{(\eta, \zeta) \in B}\left(w_{\eta \zeta, \eta \zeta}\right)_{\alpha \bar{\alpha}}-\sum_{(\beta, \gamma) \in G} \sum_{(\eta, \zeta) \in B} \frac{\left|\left(w_{\beta \gamma, \eta \zeta}\right)_{\alpha}\right|^{2}+\left|\left(w_{\beta \gamma, \eta \zeta}\right)_{\bar{\alpha}}\right|^{2}}{w_{\beta \gamma, \beta \gamma}},
$$

for each $\alpha=1, \ldots, n$. Here

$$
Q=\prod_{(\beta, \gamma) \in G} w_{\beta \gamma, \beta \gamma}
$$

Proposition 3.2. If $(n-2, n-1) \in B$, then

$$
u_{\beta \bar{\beta}} \sim 0, \quad \text { for } \beta=n-2, n-1, n .
$$

Proof. It follows from (3.4) that

$$
u_{(n-2) \overline{(n-2)}}+u_{(n-1) \overline{(n-1)}}=w_{(n-2)(n-1),(n-2)(n-1)} \sim 0 .
$$

But by (3.1) and the nonnegativity of $w_{\alpha \beta, \alpha \beta}$,

$$
u_{(n-2) \overline{(n-2)}}+u_{(n-1) \overline{(n-1)}} \geq u_{(n-2) \overline{(n-2)}}+u_{n \bar{n}} \geq 0 .
$$


Hence,

$$
u_{(n-2) \overline{(n-2)}}+u_{n \bar{n}} \sim 0 .
$$

Similarly, we also have

$$
u_{(n-1) \overline{(n-1)}}+u_{n \bar{n}} \sim 0 .
$$

The result then follows from solving the linear equations (3.7), (3.8), and (3.9).

Proposition 3.3. If $(\alpha, \beta) \in B$ for some $1 \leq \alpha<\beta \leq n-1$, then

$$
u_{\gamma \bar{\gamma}} \sim 0, \quad \text { for all } \gamma \geq \alpha
$$

Proof. By (3.4) and (3.1) we have

$$
0 \sim u_{\alpha \bar{\alpha}}+u_{\beta \bar{\beta}} \geq u_{(n-2) \overline{(n-2)}}+u_{(n-1) \overline{(n-1)}} \geq 0 .
$$

Hence,

$$
u_{(n-2) \overline{(n-2)}}+u_{(n-1) \overline{(n-1)}} \sim 0 .
$$

It follows from the proof of Proposition 3.2 that

$$
u_{\gamma \bar{\gamma}} \sim 0, \quad \text { for all } \gamma=n-2, n-1, n \text {. }
$$

But we also have that

$$
0 \sim u_{\alpha \bar{\alpha}}+u_{\beta \bar{\beta}} \geq u_{\tau \bar{\tau}}+u_{n \bar{n}} \geq 0, \quad \text { for all } \tau=\alpha, \ldots, n-1 .
$$

This implies that

$$
u_{\tau \bar{\tau}}+u_{n \bar{n}} \sim 0, \quad \text { for all } \tau=\alpha, \ldots, n-1 \text {. }
$$

Since $u_{n \bar{n}} \sim 0$, we conclude that

$$
u_{\tau \bar{\tau}} \sim 0, \quad \text { for all } \tau=\alpha, \ldots, n
$$

Let us now to prove the claim: Suppose that for some rank $r$, there exists a bad set $B$ which is none of the two cases above. Then $B$ must contain an element $\left(\alpha_{1}, \beta_{1}\right)$ such that $\beta_{1} \leq n-1$. Let

$$
\tau=\min \{\alpha \mid(\alpha, \beta) \in B \text { and } \beta \leq n-1\} .
$$

Since $B$ does not belong to Case (i), we have

$$
\{(\alpha, \beta) \mid \tau \leq \alpha<\beta \leq n\} \backslash B \neq \emptyset
$$

In other words, there exists an element $(\eta, \zeta)$ in $G$ with $\tau \leq \eta<\zeta \leq n$. But on the other hand, it follows from Proposition 3.3 that

$$
u_{\gamma \bar{\gamma}} \sim 0, \quad \text { for all } \gamma \geq \tau
$$


In particular, we have

$$
w_{\eta \zeta, \eta \zeta}=u_{\eta \bar{\eta}}+u_{\zeta \bar{\zeta}} \sim 0 .
$$

This contradicts with the first statement in Proposition 2.1. Therefore, the claim is proved.

The two cases are, respectively, settled in Lemma 3.4 and Lemma 3.5 below. We should point out that the assumption in Lemma 3.4 is weaker.

Lemma 3.4. Assume that there exists a positive integer $2 \leq \mu \leq n-2$ such that

$$
B=\{(\alpha, \beta) \mid \mu \leq \alpha<\beta \leq n\} .
$$

Denote

$$
r=N-\# B=\left(\begin{array}{l}
n \\
2
\end{array}\right)-\left(\begin{array}{c}
n-\mu+1 \\
2
\end{array}\right) .
$$

Then,

$$
\Delta \sigma_{r+1} \lesssim 0,
$$

provided that $\log f$ is Hermitian 2-concave.

Proof. Let $\phi=\sigma_{r+1}$. First, applying Proposition 3.3 to $(\mu, \mu+1) \in B$ yields that

$$
u_{\beta \bar{\beta}} \sim 0, \quad \text { for all } \beta=\mu, \ldots, n .
$$

By the Laplace equation (1.1),

$$
\sum_{\beta=1}^{\mu-1} u_{\beta \bar{\beta}} \sim f
$$

Next, it follows from (3.5) that

$$
(n-\mu) \sum_{\beta=\mu}^{n} u_{\beta \bar{\beta} \alpha} \sim 0,
$$

Here we also make use of the elementary identity

$$
\sum_{a \leq \alpha<\beta \leq b}\left(A_{\alpha}+A_{\beta}\right)=(b-a) \sum_{\beta=a}^{b} A_{\beta},
$$

for any finite numbers $\left\{A_{\beta} ; \beta=a, \ldots, b\right\}$, where $a<b$ are two positive integers. By (1.1) we obtain

$$
\sum_{\beta=1}^{\mu-1} u_{\beta \bar{\beta} \alpha} \sim f_{\alpha} .
$$

Now let us consider the second order derivatives. Apply (3.6) and (1.3) to obtain that

$$
\begin{aligned}
Q^{-1} \phi_{\alpha \bar{\alpha}} & \lesssim(n-\mu) \sum_{\gamma=\mu}^{n} u_{\alpha \bar{\alpha} \gamma \bar{\gamma}}-\sum_{\beta=1}^{\mu-1} \sum_{\gamma=\mu}^{n} \sum_{\mu \leq \tau \leq n, \tau \neq \gamma} \frac{\left|u_{\beta \bar{\tau} \alpha}\right|^{2}+\left|u_{\beta \bar{\tau} \bar{\alpha}}\right|^{2}}{u_{\beta \bar{\beta}}+u_{\gamma \bar{\gamma}}} \\
& \sim(n-\mu) \sum_{\gamma=\mu}^{n} u_{\alpha \bar{\alpha} \gamma \bar{\gamma}}-(n-\mu) \sum_{\beta=1}^{\mu-1} \sum_{\tau=\mu}^{n} \frac{\left|u_{\beta \bar{\tau} \alpha}\right|^{2}+\left|u_{\beta \bar{\tau} \bar{\alpha}}\right|^{2}}{u_{\beta \bar{\beta}}}, \quad \text { (by (3.10)). }
\end{aligned}
$$


Therefore,

$$
\begin{aligned}
\frac{\Delta \phi}{(n-\mu) Q} & \lesssim \sum_{\gamma=\mu}^{n} f_{\gamma \bar{\gamma}}-\sum_{\alpha=1}^{n} \sum_{\beta=1}^{\mu-1} \sum_{\tau=\mu}^{n} \frac{\left|u_{\beta \bar{\tau} \alpha}\right|^{2}+\left|u_{\beta \bar{\tau} \bar{\alpha}}\right|^{2}}{u_{\beta \bar{\beta}}} \\
& \left.\lesssim \sum_{\gamma=\mu}^{n} f_{\gamma \bar{\gamma}}-\sum_{\tau=\mu}^{n} \sum_{\beta=1}^{\mu-1} \frac{\left|u_{\beta \bar{\beta} \tau}\right|^{2}}{u_{\beta \bar{\beta}}} \quad \text { (by setting } \alpha=\beta \text { and dropping }\left|u_{\beta \beta \bar{\tau}}\right|^{2}\right) \\
& \lesssim \sum_{\gamma=\mu}^{n} f_{\gamma \bar{\gamma}}-\sum_{\tau=\mu}^{n}\left|\sum_{\beta=1}^{\mu-1} u_{\beta \bar{\beta} \tau}\right|^{2}\left(\sum_{\beta=1}^{\mu-1} u_{\beta \bar{\beta}}\right)^{-1} \quad \text { (by Schwarz inequality) } \\
& \sim \sum_{\gamma=\mu}^{n} f_{\gamma \bar{\gamma}}-f^{-1} \sum_{\tau=\mu}^{n}\left|f_{\tau}\right|^{2}, \quad \text { (by (3.11) and (3.13)). }
\end{aligned}
$$

Thus, the result follows immediately. $\mathrm{c}$

Lemma 3.5. Assume that

$$
B \subset\{(\alpha, n) \mid \alpha=1, \ldots, n\} .
$$

We denote by

$$
r=N-\# B
$$

Then,

$$
\Delta \sigma_{r+1} \lesssim 0
$$

provided that $f^{1 / 2}$ is Hermitian 2-concave.

Proof. In view of (3.1) and Proposition 2.1 we can assume that

$$
B=\{(\nu, n),(\nu+1, n), \ldots,(n-1, n)\}
$$

for some integer $1 \leq \nu \leq n-1$. Let

$$
\phi=\sigma_{r+1} .
$$

By (3.4) we have

$$
u_{\beta \bar{\beta}}+u_{n \bar{n}} \sim 0, \quad \beta=\nu, \ldots, n-1 .
$$

Then,

$$
u_{\nu \bar{\nu}} \sim u_{(\nu+1) \overline{(\nu+1)}} \sim \cdots \sim u_{(n-1) \overline{(n-1)}} \sim-u_{n \bar{n}} .
$$

As a consequence, for each $\gamma=\nu, \ldots, n-1$,

$$
(n-2) u_{\gamma \bar{\gamma}} \lesssim \sum_{\beta=1}^{\nu-1} u_{\beta \bar{\beta}}+(n-\nu-1) u_{\nu \bar{\nu}} \sim \sum_{\beta=1}^{n} u_{\beta \bar{\beta}}=f .
$$

Next, by (3.5) we have

$$
0 \sim \sum_{\beta=\nu}^{n-1}\left(u_{\beta \bar{\beta} \alpha}+u_{n \bar{n} \alpha}\right)=\sum_{\beta=\nu}^{n-1} u_{\beta \bar{\beta} \alpha}+(n-\nu) u_{n \bar{n} \alpha} .
$$


Thus, applying (1.1) yields that

$$
(n-\nu) \sum_{\gamma=1}^{\nu-1} u_{\gamma \bar{\gamma} \alpha}+(n-\nu-1) \sum_{\beta=\nu}^{n-1} u_{\beta \bar{\beta} \alpha} \sim(n-\nu) f_{\alpha} .
$$

For the second order derivatives, applying (1.3) to (3.6) yields that

$$
\begin{aligned}
Q^{-1} \phi_{\alpha \bar{\alpha}} \lesssim & \sum_{\beta=\nu}^{n-1} u_{\beta \bar{\beta} \alpha \bar{\alpha}}+(n-\nu) u_{n \bar{n} \alpha \bar{\alpha}} \\
& -\sum_{\beta=1}^{\nu-1} \sum_{\gamma=\nu}^{n-1} \frac{\left|u_{\beta \bar{n} \alpha}\right|^{2}+\left|u_{\beta \bar{n} \bar{\alpha}}\right|^{2}}{u_{\beta \bar{\beta}}+u_{\gamma \bar{\gamma}}}-\sum_{\beta=1}^{\nu-1} \sum_{\gamma=\nu}^{n-1} \frac{\left|u_{\beta \bar{\gamma} \alpha}\right|^{2}+\left|u_{\beta \bar{\gamma} \bar{\alpha}}\right|^{2}}{u_{\beta \bar{\beta}}+u_{n \bar{n}}} \\
& -\sum_{\nu \leq \beta<\gamma \leq n-1} \frac{\left|u_{\beta \bar{n} \alpha}\right|^{2}+\left|u_{\beta \bar{n} \bar{\alpha}}\right|^{2}+\left|u_{\gamma \bar{n} \alpha}\right|^{2}+\left|u_{\gamma \bar{n} \bar{\alpha}}\right|^{2}}{u_{\beta \bar{\beta}}+u_{\gamma \bar{\gamma}}}
\end{aligned}
$$

Thus,

$$
\begin{aligned}
Q^{-1} \Delta \phi & \lesssim \sum_{\beta=\nu}^{n-1} f_{\beta \bar{\beta}}+(n-\nu) f_{n \bar{n}}-\sum_{\beta=1}^{\nu-1} \sum_{\gamma=\nu}^{n-1} \frac{\left|u_{\beta \bar{\beta}}\right|^{2}}{u_{\beta \bar{\beta}}+u_{\gamma \bar{\gamma}}} \\
& -\sum_{\beta=1}^{\nu-1} \sum_{\gamma=\nu}^{n-1} \frac{\left|u_{\beta \bar{\beta} \gamma}\right|^{2}}{u_{\beta \bar{\beta}}+u_{n \bar{n}}}-\sum_{\nu \leq \beta<\gamma \leq n-1} \frac{\left|u_{\beta \bar{\beta} n}\right|^{2}+\left|u_{n \bar{n} \beta}\right|^{2}+\left|u_{\gamma \bar{\gamma} n}\right|^{2}+\left|u_{n \bar{n} \gamma}\right|^{2}}{u_{\beta \bar{\beta}}+u_{\gamma \bar{\gamma}}} \\
& =(n-\nu) f_{n \bar{n}}-\sum_{\beta=1}^{\nu-1} \sum_{\gamma=\nu}^{n-1} \frac{\left|u_{\beta \bar{\beta} n}\right|^{2}}{u_{\beta \bar{\beta}}+u_{\gamma \bar{\gamma}}}-\sum_{\nu \leq \beta \neq \gamma \leq n-1} \frac{\left|u_{\beta \bar{\beta} n}\right|^{2}}{u_{\beta \bar{\beta}}+u_{\gamma \bar{\gamma}}} \\
& +\sum_{\gamma=\nu}^{n-1}\left(f_{\gamma \bar{\gamma}}-\sum_{\beta=1}^{\nu-1} \frac{\left|u_{\beta \bar{\beta} \gamma}\right|^{2}}{u_{\beta \bar{\beta}}+u_{n \bar{n}}}-\sum_{\nu \leq \beta \leq n-1, \beta \neq \gamma} \frac{\left|u_{n \bar{n} \gamma}\right|^{2}}{u_{\beta \bar{\beta}}+u_{\gamma \bar{\gamma}}}\right)
\end{aligned}
$$

Apply the Schwarz inequality to the last two terms in (3.18) to obtain that

$$
\begin{aligned}
& \sum_{\beta=1}^{\nu-1} \sum_{\gamma=\nu}^{n-1} \frac{\mid u_{\beta \bar{\beta}} n}{u_{\beta \bar{\beta}}+u_{\gamma \bar{\gamma}}}+\sum_{\beta=\nu}^{n-1} \sum_{\nu \leq \gamma \leq n-1, \gamma \neq \beta} \frac{\left|u_{\beta \bar{\beta}} n\right|^{2}}{u_{\beta \bar{\beta}}+u_{\gamma \bar{\gamma}}} \\
& \geq \frac{\left|(n-\nu) \sum_{\beta=1}^{\nu-1} u_{\beta \bar{\beta} n}+(n-\nu-1) \sum_{\beta=\nu}^{n-1} u_{\beta \bar{\beta} n}\right|^{2}}{(n-\nu) \sum_{\beta=1}^{\nu-1} u_{\beta \bar{\beta}}+(2 n-\nu-3) \sum_{\beta=\nu}^{n-1} u_{\beta \bar{\beta}}} .
\end{aligned}
$$

By (3.14) and (3.15) we have

$$
\begin{gathered}
(n-\nu) \sum_{\beta=1}^{\nu-1} u_{\beta \bar{\beta}}+(2 n-\nu-3) \sum_{\beta=\nu}^{n-1} u_{\beta \bar{\beta}} \sim(n-\nu) f+(n-\nu)(n-2) u_{\nu \bar{\nu}} \\
\lesssim 2(n-\nu) f .
\end{gathered}
$$

Plugging this and (3.17) into (3.20) yields that

$$
\sum_{\beta=1}^{\nu-1} \sum_{\gamma=\nu}^{n-1} \frac{\left|u_{\beta \bar{\beta} n}\right|^{2}}{u_{\beta \bar{\beta}}+u_{\gamma \bar{\gamma}}}+\sum_{\nu \leq \beta \neq \gamma \leq n-1} \frac{\left|u_{\beta \bar{\beta} n}\right|^{2}}{u_{\beta \bar{\beta}}+u_{\gamma \bar{\gamma}}} \geq \frac{(n-\nu)\left|f_{n}\right|^{2}}{2 f} .
$$


We next to handle (3.19). For each $\gamma=\nu, \ldots, n-1$, we apply the Schwarz inequality to obtain that

$$
\begin{aligned}
& \sum_{\beta=1}^{\nu-1} \frac{\left|u_{\beta \bar{\beta} \gamma}\right|^{2}}{u_{\beta \bar{\beta}}+u_{n \bar{n}}}+\sum_{\nu \leq \beta \leq n-1, \beta \neq \gamma} \frac{\left|u_{n \bar{n} \gamma}\right|^{2}}{u_{\beta \bar{\beta}}+u_{\gamma \bar{\gamma}}} \\
& \geq \frac{\left|\sum_{\beta=1}^{\nu-1} u_{\beta \bar{\beta} \gamma}-(n-\nu-1) u_{n \bar{n} \gamma}\right|^{2}}{\sum_{\beta=1}^{\nu-1} u_{\beta \bar{\beta}}+(\nu-1) u_{n \bar{n}}+\sum_{\nu \leq \beta \leq n-1} u_{\beta \bar{\beta}}+(n-\nu-2) u_{\gamma \bar{\gamma}}} \\
& \gtrsim \frac{\left|f_{\gamma}\right|^{2}}{2 \sum_{\beta=1}^{\nu-1} u_{\beta \bar{\beta}}+2(n-\nu-1) u_{\nu \bar{\nu}}} \quad(\text { by }(3.16),(3.1), \text { and }(3.14)) \\
& \sim \frac{\left|f_{\gamma}\right|^{2}}{2 f}, \quad(\text { by }(3.15)) .
\end{aligned}
$$

Now substituting this and (3.21) into (3.19) and (3.18), respectively, yields that

$$
\begin{aligned}
Q^{-1} \Delta \phi & \lesssim \sum_{\gamma=\nu}^{n-1}\left(f_{\gamma \bar{\gamma}}-(2 f)^{-1}\left|f_{\gamma}\right|^{2}\right)+(n-\nu)\left(f_{n \bar{n}}-(2 f)^{-1}\left|f_{n}\right|^{2}\right) \\
& =\sum_{\gamma=\nu}^{n-1}\left[\left(f_{\gamma \bar{\gamma}}-(2 f)^{-1}\left|f_{\gamma}\right|^{2}\right)+\left(f_{n \bar{n}}-(2 f)^{-1}\left|f_{n}\right|^{2}\right)\right] .
\end{aligned}
$$

Therefore, if $f^{1 / 2}$ is Hermitian 2-concave then we conclude that $\Delta \phi \lesssim 0$.

Hence, the proof of Theorem 3.1 is completed.

4. General case. In this section, we shall prove Theorem 1.1 for any $k=$ $1, \ldots, n-1$. As in the previous section, we only have to show (2.2) at a point $x$ near $x_{0}$. Let us choose a coordinate system at $x$ so that

$$
u_{\alpha \bar{\beta}}=\delta_{\alpha \beta} u_{\alpha \bar{\alpha}},
$$

and

$$
u_{1 \overline{1}} \geq u_{2 \overline{2}} \geq \cdots \geq u_{n \bar{n}} .
$$

Then the $w$-matrix is also diagonalized:

$$
w_{\alpha_{1} \ldots \alpha_{k}, \beta_{1} \ldots \beta_{k}}=\left(\prod_{i=1}^{k} \delta_{\alpha_{i} \beta_{i}}\right) w_{\alpha_{1} \ldots \alpha_{k}, \alpha_{1} \ldots \alpha_{k}}=\prod_{i=1}^{k} \delta_{\alpha_{i} \beta_{i}} \sum_{i=1}^{k} u_{\alpha_{i} \bar{\alpha}_{i}} .
$$

But in general we do not have a similar order for $w_{\alpha_{1} \ldots \alpha_{k}, \alpha_{1} \ldots \alpha_{k}}$ in terms of the indices. Recall that the rank of $w$-matrix is assumed to be $r$ at $x_{0}$. We can pick a constant $c>0$ so that the $w$-matrix has exactly $r$ eigenvalues $\geq c$ near $x_{0}$. We denote by

$$
G=\left\{\left(\alpha_{1}, \ldots, \alpha_{k}\right) \mid w_{\alpha_{1} \ldots \alpha_{k}, \alpha_{1} \ldots \alpha_{k}} \geq c, 1 \leq \alpha_{1}<\cdots<\alpha_{k} \leq n\right\},
$$

and

$$
B=\left\{\left(\alpha_{1}, \ldots, \alpha_{k}\right) \mid 1 \leq \alpha_{1}<\cdots<\alpha_{k} \leq n\right\} \backslash G .
$$

Then, $\# G=r$ and $\# B=N-r$. As before, $G$ and $B$ are referred to be the good set and bad set of indices, respectively.

We claim that any bad set must belong to one of the two cases. 
1. There exists a positive integer $\mu \leq n-k$ so that

$$
B=\left\{\left(\alpha_{1}, \ldots, \alpha_{k}\right) \mid \mu \leq \alpha_{1}<\alpha_{2}<\cdots<\alpha_{k} \leq n\right\} .
$$

2. There exist positive integers $\nu \leq n-k$ and $m \leq k-1$ so that

$$
B=\left\{\left(\alpha_{1}, \ldots, \alpha_{k-m}, n-m+1, \ldots, n\right) \mid \nu \leq \alpha_{1}<\cdots<\alpha_{k-m} \leq n-m\right\} .
$$

Equivalently, one can also think that Case (2) consists of the following $k-1$ smaller cases in terms of the integer $m$. Namely, for each $m=1, \ldots, k-1$, Case $(2: m)$ is that there exists a positive integer $\nu_{m} \leq n-k$ so that

$$
B=\left\{\left(\alpha_{1}, \ldots, \alpha_{k-m}, n-m+1, \ldots, n\right) \mid \nu_{m} \leq \alpha_{1}<\cdots<\alpha_{k-m} \leq n-m\right\} .
$$

Assuming this claim, we only need to verify (2.2) for these two cases. Before proving this claim, let us prove some simple facts. Our starting point is (2.8) in Proposition 2.2.

Proposition 4.1. Let $A=\left(a_{p q}\right)_{1 \leq p, q \leq l}, l \geq 2$, be a matrix given by

$$
a_{p q}=0, \quad \text { for all } p+q=l+1, \quad \text { and } a_{p q}=1 \text { for all else } p, q .
$$

Then $A$ is invertible, and $A^{-1}=(l-1)^{-1}\left(b_{p q}\right)$ in which

$$
b_{p q}=2-l, \quad \text { for all } p+q=l+1, \quad \text { and } b_{p q}=1 \text { for else. }
$$

Proof. The result follows from a direct verification.

Proposition 4.2. Suppose that $w_{(n-k) \ldots(n-1),(n-k) \ldots(n-1)} \sim 0$. Then

$$
u_{\beta \bar{\beta}} \sim 0, \quad \text { for all } \beta=n-k, \ldots, n \text {. }
$$

Proof. By (4.1) we have

$$
w_{(n-k) \ldots(n-1),(n-k) \ldots(n-1)}=\sum_{\beta=n-k}^{n-1} u_{\beta \bar{\beta}} \geq \sum_{i=1}^{k} u_{\gamma_{i} \bar{\gamma}_{i}}=w_{\gamma_{1} \ldots \gamma_{k}, \gamma_{1} \ldots \gamma_{k}} \geq 0,
$$

for all $n-k \leq \gamma_{1}<\cdots<\gamma_{k} \leq n$. Hence,

$$
\sum_{i=1}^{k} u_{\gamma_{i} \bar{\gamma}_{i}} \sim 0, \quad \text { for all } n-k \leq \gamma_{1}<\cdots<\gamma_{k} \leq n .
$$

Then, the result follows from solving these linear equations. Indeed, we can rewrite (4.2) as

$$
A U \sim 0
$$

Here $U$ is the transpose of $\left(u_{(n-k) \overline{(n-k)}}, \ldots, u_{n \bar{n}}\right)$, and $A=\left(a_{p q}\right)$ is the $k+1$ by $k+1$ matrix given by

$$
a_{p q}=0, \quad \text { for all } p+q=k+2, \quad \text { and } a_{p q}=1 \text { for all else } 1 \leq p, q \leq k+1 .
$$

Then $A$ is invertible by Proposition 4.1. 
Proposition 4.3. If there exists a $\left(\beta_{1}, \ldots, \beta_{k}\right) \in B$ with $\beta_{k} \leq n-1$, then

$$
u_{\gamma \bar{\gamma}} \sim 0, \quad \text { for all } \gamma \geq \beta_{1} .
$$

Proof. By (2.8) we know that

$$
w_{\beta_{1} \ldots \beta_{k}, \beta_{1} \ldots \beta_{k}} \sim 0
$$

In view of (4.1), we have

$$
0 \leq w_{(n-k) \ldots(n-1),(n-k) \ldots(n-1)} \leq w_{\beta_{1} \ldots \beta_{k}, \beta_{1} \ldots \beta_{k}} .
$$

Thus,

$$
w_{(n-k) \ldots(n-1),(n-k) \ldots(n-1)} \sim 0 .
$$

It follows from Proposition 4.2 that

$$
u_{\gamma \bar{\gamma}} \sim 0, \quad \text { for all } \gamma=n-k, \ldots, n .
$$

For each $\zeta$ such that $\beta_{1} \leq \zeta \leq n-k$, we also have

$$
w_{\zeta(n-k+2) \ldots n, \zeta(n-k+2) \ldots n} \sim 0,
$$

since

$$
0 \leq w_{\zeta(n-k+2) \ldots n, \zeta(n-k+2) \ldots n} \leq w_{\beta_{1} \ldots \beta_{k}, \beta_{1} \ldots \beta_{k}} .
$$

Hence,

$$
0 \sim u_{\zeta \bar{\zeta}}+\sum_{\gamma=n-k+2}^{n} u_{\gamma \bar{\gamma}} \sim u_{\zeta \bar{\zeta}}
$$

This completes the proof. $\square$

Proposition 4.4. Let $m$ be an integer such that $1 \leq m \leq k-1$. Suppose that there exists a $\left(\beta_{1}, \ldots, \beta_{k-m}, n-m+1, \ldots, n\right) \in B$ with $\beta_{k-m} \leq n-m-1$. Then

$$
u_{\gamma \bar{\gamma}} \sim-\frac{1}{k-m} \sum_{\zeta=n-m+1}^{n} u_{\zeta \bar{\zeta}}, \quad \text { for all } \beta_{1} \leq \gamma \leq n-m .
$$

As a consequence,

$$
w_{\gamma_{1} \ldots \gamma_{k-m}(n-m+1) \ldots n, \gamma_{1} \ldots \gamma_{k-m}(n-m+1) \ldots n} \sim 0,
$$

for all $\beta_{1} \leq \gamma_{1}<\cdots<\gamma_{k-m} \leq n-m$.

Proof. By (2.8) we have

$$
w_{\beta_{1} \ldots \beta_{k-m}(n-m+1) \ldots n, \beta_{1} \ldots \beta_{k-m}(n-m+1) \ldots n} \sim 0 .
$$

Note that $\beta_{k-m} \leq n-m-1$ implies that

$$
\beta_{i} \leq n-k+i-1, \quad \text { for each } i=1, \ldots, k-m .
$$


Then, by (4.1),

$$
\begin{aligned}
& w_{\beta_{1} \ldots \beta_{k-m}(n-m+1) \ldots n, \beta_{1} \ldots \beta_{k-m}(n-m+1) \ldots n} \\
& \quad \geq w_{\gamma_{1} \ldots \gamma_{k-m}}(n-m+1) \ldots n, \gamma_{1} \ldots \gamma_{k-m}(n-m+1) \ldots n \\
& \quad \geq 0,
\end{aligned}
$$

for all $n-k \leq \gamma_{1}<\cdots<\gamma_{k-m} \leq n-m$. This implies that

$$
w_{\gamma_{1} \ldots \gamma_{k-m}(n-m+1) \ldots n, \gamma_{1} \ldots \gamma_{k-m}(n-m+1) \ldots n} \sim 0,
$$

for all $n-k \leq \gamma_{1}<\cdots<\gamma_{k-m} \leq n-m$. Thus,

$$
\sum_{i=1}^{k-m} u_{\gamma_{i} \bar{\gamma}_{i}} \sim-\sum_{\zeta=n-m+1}^{n} u_{\zeta \bar{\zeta}}
$$

for all $n-k \leq \gamma_{1}<\cdots<\gamma_{k-m} \leq n-m$. Let us solve these linear equations: We can reformulate the linear equations (4.4) as

$$
A U \sim B,
$$

Here $U$ is the transpose of $\left(u_{(n-k) \overline{(n-k)}}, \ldots, u_{(n-m)} \overline{(n-m)}\right), A=\left(a_{p q}\right)$ is the $(k-m+1)$ by $(k-m+1)$ matrix given by

$$
a_{p q}= \begin{cases}0, & \text { if } p+q=k+m+2, \quad 1 \leq p, q \leq k-m+1, \\ 1, & \text { otherwise }\end{cases}
$$

and $B$ is the transpose of the following $(k-m+1)$-vector

$$
-\sum_{\zeta=n-m+1}^{n} u_{\zeta \bar{\zeta}} \cdot(1, \ldots, 1) .
$$

Now applying Proposition 4.1 to (4.5) yields that

$$
u_{\gamma \bar{\gamma}} \sim-\frac{1}{k-m} \sum_{\zeta=n-m+1}^{n} u_{\zeta \bar{\zeta}}, \quad \text { for all } n-k \leq \gamma \leq n-m .
$$

For those $\gamma$ with $\beta_{1} \leq \gamma \leq n-k$, we have by (4.1) and (4.3) that

$$
0 \leq w_{\gamma(n-k+2) \ldots n, \gamma(n-k+2) \ldots n} \leq w_{\beta_{1} \ldots \beta_{k-m}(n-m+1) \ldots n, \beta_{1} \ldots \beta_{k-m}(n-m+1) \ldots n} .
$$

Thus,

$$
\begin{aligned}
0 & \sim w_{\gamma(n-k+2) \ldots n, \gamma(n-k+2) \ldots n} \\
& \sim u_{\gamma \bar{\gamma}}+\sum_{\eta=n-k+2}^{n-m} u_{\eta \bar{\eta}}+\sum_{\zeta=n-m+1}^{n} u_{\zeta \bar{\zeta}} \\
& \sim u_{\gamma \bar{\gamma}}+\frac{1}{k-m} \sum_{\zeta=n-m+1}^{n} u_{\zeta \bar{\zeta}}, \quad(\text { by }(4.6)) .
\end{aligned}
$$

Therefore, we have shown that

$$
u_{\gamma \bar{\gamma}} \sim-\frac{1}{k-m} \sum_{\zeta=n-m+1}^{n} u_{\zeta \bar{\zeta}}, \quad \text { for all } \gamma=\beta_{1}, \ldots, n-m .
$$


COROLlary 4.5. Suppose that there exist two positive integers $\nu$ and $m, \nu \leq$ $n-k, m \leq k-1$, such that

$$
B=\left\{\left(\alpha_{1}, \ldots, \alpha_{k-m}, n-m+1, \ldots, n\right) \mid \nu \leq \alpha_{1}<\cdots<\alpha_{k-m} \leq n-m\right\} .
$$

Then,

$$
u_{\gamma \bar{\gamma}} \sim-\frac{1}{k-m} \sum_{\zeta=n-m+1}^{n} u_{\zeta \bar{\zeta}}, \quad \text { for all } \gamma=\nu, \ldots, n-m
$$

Proof. Applying Proposition 4.4 to

$$
(\nu, n-k+1, \ldots, n-m-1, n-m+1, \ldots, n) \in B
$$

yields the desired result. $\square$

Let us prove this claim. Let $B$ be a bad set which does not belong to Case (1) nor Case (2). The idea is to show that there exists an element in $G$ which $\sim 0$. Then this would contradicts with the first statement in Proposition 2.1.

First, assume that $B$ contains an element $\left(\beta_{1}, \ldots, \beta_{k}\right)$ with $\beta_{k} \leq n-1$. Let

$$
\tau_{1}=\min \left\{\alpha_{1} \mid\left(\alpha_{1}, \ldots, \alpha_{k}\right) \in B, \alpha_{k} \leq n-1\right\} .
$$

Since $B$ does not belong to Case (1), we have

$$
\left\{\left(\alpha_{1}, \ldots, \alpha_{k}\right) \mid \tau_{1} \leq \alpha_{1}<\cdots<\alpha_{k} \leq n\right\} \backslash B \neq \emptyset .
$$

In other words, there exists a $\left(\eta_{1}, \ldots, \eta_{k}\right) \in G$ with $\tau_{1} \leq \eta_{1}<\cdots<\eta_{k} \leq n$. But on the other hand, we have by Proposition 4.3 that

$$
u_{\gamma \bar{\gamma}} \sim 0, \quad \text { for all } \gamma \geq \tau_{1} .
$$

In particular,

$$
w_{\eta_{1} \ldots \eta_{k}, \eta_{1} \ldots \eta_{k}}=\sum_{i=1}^{k} u_{\eta_{i} \bar{\eta}_{i}} \sim 0
$$

This contradicts with Proposition 2.1. Thus, if $B$ does not belong to Case (1) nor Case (2), then $B$ must be a subset of

$$
\left\{\left(\alpha_{1}, \ldots, \alpha_{k-1}, n\right) \mid 1 \leq \alpha_{1}<\cdots<\alpha_{k-1} \leq n-1\right\} .
$$

Now suppose that $B$ is a subset of

$$
\left\{\left(\alpha_{1}, \ldots, \alpha_{k-l}, n-l+1, \ldots, n\right) \mid 1 \leq \alpha_{1} \leq \cdots \leq \alpha_{k-l} \leq n-l\right\},
$$

for some $1 \leq l \leq k-2$, and that $B$ contains an element $\left(\gamma_{1}, \ldots, \gamma_{k-1}, n-l+1, \ldots, n\right)$ with $\gamma_{k-1} \leq n-l-1$. Let

$$
\lambda=\min \left\{\alpha_{1} \mid\left(\alpha_{1}, \ldots, \alpha_{k-l}, n-l+1, \ldots, n\right) \in B, \alpha_{k-l} \leq n-l-1\right\} .
$$


Since $B$ does not belong to Case $(2: l)$, we have

$$
\left\{\left(\alpha_{1}, \ldots, \alpha_{k-l}, n-l+1, \ldots, n\right) \mid \lambda \leq \alpha_{1}<\cdots<\alpha_{k-l} \leq n-l\right\} \backslash B \neq \emptyset .
$$

Thus, there exists a $\left(\zeta_{1}, \ldots, \zeta_{k-l}, n-l+1, \ldots, n\right)$ in $G$ such that

$$
\lambda \leq \zeta_{1}<\cdots<\zeta_{k-l} \leq n-l .
$$

But it follows from Proposition 4.4 that

$$
w_{\zeta_{1} \ldots \zeta_{k-l}(n-l+1) \ldots n, \zeta_{1} \ldots \zeta_{k-l}(n-l+1) \ldots n} \sim 0 .
$$

This contradicts with Proposition 2.1. Thus, $B$ is indeed a subset of

$$
\left\{\left(\alpha_{1}, \ldots, \alpha_{k-l-1}, n-l, \ldots, n\right) \mid 1 \leq \alpha_{1} \leq \cdots \leq \alpha_{k-l-1} \leq n-l-1\right\},
$$

Therefore, by induction, we obtain that $B$ is a subset of

$$
\left\{\left(\alpha_{1}, n-k+2, \ldots, n\right) \mid 1 \leq \alpha_{1} \leq n-k+1\right\} .
$$

This implies that $B$ belongs to Case $(2: k-1)$. It is a contradiction. This proves the claim.

We treat Case (1) and Case (2) in the following Lemma 4.6 and Lemma 4.7, respectively. Thus, the proof of Theorem 1.1 is completed.

Lemma 4.6. Assume that there exists an integer $\mu \in\{2, \ldots, n-k\}$ so that

$$
B=\left\{\left(\alpha_{1}, \ldots, \alpha_{k}\right) \mid \mu \leq \alpha_{1}<\cdots \alpha_{k} \leq n\right\} .
$$

Let

$$
r=\left(\begin{array}{l}
n \\
k
\end{array}\right)-\# B=\left(\begin{array}{l}
n \\
k
\end{array}\right)-\left(\begin{array}{c}
n-\mu+1 \\
k
\end{array}\right)
$$

Then,

$$
\Delta \sigma_{r+1} \lesssim 0
$$

provided that $\log f$ is Hermitian $k$-concave in $\Omega$.

Proof. Let $\phi=\sigma_{r+1}$. Apply Proposition 4.3 to $(\mu, n-k+1, \ldots, n-1) \in B$ to obtain that

$$
u_{\gamma \bar{\gamma}} \sim 0, \quad \text { for all } \gamma \geq \mu \text {. }
$$

Then,

$$
\sum_{\gamma=1}^{\mu-1} u_{\gamma \bar{\gamma}} \sim \sum_{\gamma=1}^{n} u_{\gamma \bar{\gamma}}=f .
$$

It follows from (2.9) that, for each $\alpha=1, \ldots, n$,

$$
\left(\begin{array}{c}
n-\mu \\
k-1
\end{array}\right) \sum_{\beta=\mu}^{n} u_{\beta \bar{\beta} \alpha}=\sum_{\mu \leq \beta_{1}<\cdots<\beta_{k} \leq n}\left(u_{\beta_{1} \bar{\beta}_{1} \alpha}+\cdots+u_{\beta_{k} \bar{\beta}_{k} \alpha}\right) \sim 0 .
$$


Then,

$$
\sum_{\gamma=1}^{\mu-1} u_{\gamma \bar{\gamma} \alpha} \sim \sum_{\gamma=1}^{n} u_{\gamma \bar{\gamma} \alpha}=f_{\alpha}, \quad \text { for all } \alpha=1, \ldots, n .
$$

By (2.10) we have

$$
\begin{aligned}
Q^{-1} \phi_{\alpha \bar{\alpha}} \lesssim & \sum_{\left(\beta_{1}, \ldots, \beta_{k}\right) \in B}\left(w_{\beta_{1} \ldots \beta_{k}, \beta_{1} \ldots \beta_{k}}\right)_{\alpha \bar{\alpha}} \\
& -\sum_{\left(\gamma_{1}, \ldots, \gamma_{k}\right) \in G} \sum_{\left(\beta_{1}, \ldots, \beta_{k}\right) \in B} \frac{\left|\left(w_{\beta_{1} \ldots \beta_{k}, \gamma_{1} \ldots \gamma_{k}}\right)_{\alpha}\right|^{2}+\left|\left(w_{\beta_{1} \ldots \beta_{k}, \gamma_{1} \ldots \gamma_{k}}\right)_{\bar{\alpha}}\right|^{2}}{w_{\gamma_{1} \ldots \gamma_{k}, \gamma_{1} \ldots \gamma_{k}}}
\end{aligned}
$$

Note that

$$
\begin{aligned}
\sum_{\left(\beta_{1}, \ldots, \beta_{k}\right) \in B}\left(w_{\beta_{1} \ldots \beta_{k}, \beta_{1} \ldots \beta_{k}}\right)_{\alpha \bar{\alpha}} & =\sum_{\mu \leq \beta_{1}<\cdots<\beta_{k} \leq n}\left(u_{\beta_{1} \bar{\beta}_{1} \alpha \bar{\alpha}}+\cdots+u_{\beta_{k} \bar{\beta}_{k} \alpha \bar{\alpha}}\right) \\
& =\left(\begin{array}{c}
n-\mu \\
k-1
\end{array}\right) \sum_{\beta=\mu}^{n} u_{\beta \bar{\beta} \alpha \bar{\alpha}} .
\end{aligned}
$$

Recall that $w_{\beta_{1} \ldots \beta_{k}, \gamma_{1} \ldots \gamma_{k}}$ is identically zero as long as $\left\{\beta_{1}, \ldots, \beta_{k}\right\}$ and $\left\{\gamma_{1}, \ldots, \gamma_{k}\right\}$ are differed by two elements. We then obtain that

$$
\begin{aligned}
& \sum_{\left(\gamma_{1}, \ldots, \gamma_{k}\right) \in G} \sum_{\left(\beta_{1}, \ldots, \beta_{k}\right) \in B} \frac{\left.\left|\left(w_{\beta_{1} \ldots \beta_{k}, \gamma_{1} \ldots \gamma_{k}}\right)_{\alpha}\right|^{2}+\mid w_{\beta_{1} \ldots \beta_{k}, \gamma_{1} \ldots \gamma_{k}}\right)\left._{\bar{\alpha}}\right|^{2}}{w_{\gamma_{1} \ldots \gamma_{k}, \gamma_{1} \ldots \gamma_{k}}} \\
= & \sum_{\gamma=1}^{\mu-1} \sum_{\left(\beta_{1}, \ldots, \beta_{k}\right) \in B} \sum_{j=1}^{k} \frac{\left|u_{\beta_{j} \bar{\gamma} \alpha}\right|^{2}+\left|u_{\beta_{j} \bar{\gamma} \bar{\alpha}}\right|^{2}}{u_{\gamma \bar{\gamma}}+u_{\beta_{1} \bar{\beta}_{1}}+\cdots+\widehat{u_{\beta_{j} \bar{\beta}_{j}}}+\cdots+u_{\beta_{k} \bar{\beta}_{k}}} \\
\sim & \left(\begin{array}{l}
n-\mu \\
k-1
\end{array}\right) \sum_{\gamma=1}^{\mu-1} \sum_{\beta=\mu}^{n} \frac{\left|u_{\beta \bar{\gamma} \alpha}\right|^{2}+\left|u_{\beta \bar{\gamma} \bar{\alpha}}\right|^{2}}{u_{\gamma \bar{\gamma}}}, \quad \text { (by (4.8)). }
\end{aligned}
$$

Here we use the convention: $a_{1}+\cdots+\widehat{a_{j}}+\cdots+a_{k}$ means that the term $a_{j}$ is omitted in the summation. Then, we have

$$
\begin{aligned}
Q^{-1} \Delta \phi & =Q^{-1} \sum_{\alpha=1}^{n} \phi_{\alpha \bar{\alpha}} \\
& \lesssim\left(\begin{array}{c}
n-\mu \\
k-1
\end{array}\right) \sum_{\beta=\mu}^{n} \sum_{\alpha=1}^{n} u_{\beta \bar{\beta} \alpha \bar{\alpha}}-\left(\begin{array}{c}
n-\mu \\
k-1
\end{array}\right) \sum_{\alpha=1}^{n} \sum_{\gamma=1}^{\mu-1} \sum_{\beta=\mu}^{n} \frac{\left|u_{\beta \bar{\gamma} \alpha}\right|^{2}+\left|u_{\beta \bar{\gamma} \bar{\alpha}}\right|^{2}}{u_{\gamma \bar{\gamma}}} \\
& \lesssim\left(\begin{array}{l}
n-\mu \\
k-1
\end{array}\right)\left(\sum_{\beta=\mu}^{n} f_{\beta \bar{\beta}}-\sum_{\beta=\mu}^{n} \sum_{\gamma=1}^{\mu-1} \frac{\left|u_{\gamma \bar{\gamma} \beta}\right|^{2}}{u_{\gamma \bar{\gamma}}}\right) \\
& \lesssim\left(\begin{array}{l}
n-\mu \\
k-1
\end{array}\right) \sum_{\beta=\mu}^{n}\left[f_{\beta \bar{\beta}}-\left|\sum_{\gamma=1}^{\mu-1} u_{\gamma \bar{\gamma} \beta}\right|^{2}\left(\sum_{\gamma=1}^{\mu-1} u_{\gamma \bar{\gamma}}\right)^{-1}\right] \\
& \sim\left(\begin{array}{l}
n-\mu \\
k-1
\end{array}\right) \sum_{\beta=\mu}^{n}\left(f_{\beta \bar{\beta}}-\left|f_{\beta}\right|^{2} f^{-1}\right), \quad \text { (by (4.9) and (4.10)). }
\end{aligned}
$$


Therefore, $\Delta \phi \lesssim 0$ if $\log f$ is Hermitian $k$-concave in $\Omega$. $\square$

Lemma 4.7. Assume that there exist positive integers $m \leq k-1$ and $\nu \leq n-k$ so that

$$
B=\left\{\left(\alpha_{1}, \ldots, \alpha_{k-m}, n-m+1, \ldots, n\right) \mid \nu \leq \alpha_{1}<\cdots<\alpha_{k-m} \leq n-m\right\} .
$$

Let

$$
r=\left(\begin{array}{l}
n \\
k
\end{array}\right)-\# B=\left(\begin{array}{l}
n \\
k
\end{array}\right)-\left(\begin{array}{c}
n-m-\nu+1 \\
k-m
\end{array}\right)
$$

Then,

$$
\Delta \sigma_{r+1} \lesssim 0
$$

provided that the matrix $\left(k f f_{\alpha \bar{\beta}}-f_{\alpha} f_{\bar{\beta}}\right)_{1 \leq \alpha, \beta \leq n}$ is $k$-concave in $\Omega$, equivalently, $f^{1-1 / k}$ is Hermitian $k$-concave for $k \geq 2$, and $\log f$ is Hermitian 1 -concave when $k=1$.

Proof. Let $\phi=\sigma_{r+1}$. Notice that, by (4.7) and (4.1),

$$
\begin{aligned}
f & =\sum_{\gamma=1}^{\nu-1} u_{\gamma \bar{\gamma}}+\sum_{\eta=\nu}^{n-m} u_{\eta \bar{\eta}}+\sum_{\zeta=n-m+1}^{n} u_{\zeta \bar{\zeta}} \\
& \sim \sum_{\gamma=1}^{\nu-1} u_{\gamma \bar{\gamma}}+(n-k-\nu+1) u_{\nu \bar{\nu}} \\
& \gtrsim(n-k) u_{\nu \bar{\nu}} .
\end{aligned}
$$

It follows from (2.9) that

$$
\begin{aligned}
& \left(\begin{array}{l}
n-m-\nu \\
k-m-1
\end{array}\right) \sum_{\eta=\nu}^{n-m} u_{\eta \bar{\eta} \alpha}+\left(\begin{array}{c}
n-m-\nu+1 \\
k-m
\end{array}\right) \sum_{\zeta=n-m+1}^{n} u_{\zeta \bar{\zeta} \alpha} \\
& =\sum_{\nu \leq \beta_{1}<\cdots<\beta_{k} \leq n-m}\left(\sum_{i=1}^{k-m} u_{\beta_{i} \bar{\beta}_{i} \alpha}+\sum_{\zeta=n-m+1}^{n} u_{\zeta \bar{\zeta} \alpha}\right) \sim 0,
\end{aligned}
$$

for each $\alpha=1, \ldots, n$. Then, for each $\alpha$,

$$
\begin{aligned}
& \left(\begin{array}{c}
n-m-\nu+1 \\
k-m
\end{array}\right) f_{\alpha}=\left(\begin{array}{c}
n-m-\nu+1 \\
k-m
\end{array}\right) \sum_{\gamma=1}^{n} u_{\gamma \bar{\gamma} \alpha} \\
& \sim\left(\begin{array}{c}
n-m-\nu+1 \\
k-m
\end{array}\right) \sum_{\gamma=1}^{\nu-1} u_{\gamma \bar{\gamma} \alpha}+\left(\begin{array}{c}
n-m-\nu \\
k-m
\end{array}\right) \sum_{\eta=\nu}^{n-m} u_{\eta \bar{\eta} \alpha}
\end{aligned}
$$

where we make use of the elementary identity

$$
\left(\begin{array}{l}
p \\
q
\end{array}\right)=\left(\begin{array}{c}
p-1 \\
q
\end{array}\right)+\left(\begin{array}{l}
p-1 \\
q-1
\end{array}\right)
$$


for any two positive integers $q$ and $p \geq 2$. Similarly, we have

$$
\begin{aligned}
& \left(\begin{array}{c}
n-m-\nu \\
k-m-1
\end{array}\right) f_{\alpha} \\
& \quad \sim\left(\begin{array}{c}
n-m-\nu \\
k-m-1
\end{array}\right) \sum_{\gamma=1}^{\nu-1} u_{\gamma \bar{\gamma} \eta}-\left(\begin{array}{c}
n-m-\nu \\
k-m
\end{array}\right) \sum_{\zeta=n-m+1}^{n} u_{\zeta \bar{\zeta} \alpha}
\end{aligned}
$$

for each $\alpha=1, \ldots, n$.

By (2.10) we have

$$
\begin{aligned}
& Q^{-1} \phi_{\alpha \bar{\alpha}} \lesssim \sum_{\nu \leq \beta_{1}<\cdots<\beta_{k-m} \leq n-m}\left(w_{\beta_{1} \ldots \beta_{k-m}(n-m+1) \ldots n, \beta_{1} \ldots \beta_{k-m}(n-m+1) \ldots n}\right)_{\alpha \bar{\alpha}} \\
& -\sum_{\left(\gamma_{1}, \ldots, \gamma_{k}\right) \in G \leq \beta_{1}<\cdots<\beta_{k-m} \leq n-m} \frac{\left|\left(w_{\beta_{1} \ldots \beta_{k-m}(n-m+1) \ldots n, \gamma_{1} \ldots \gamma_{k}}\right)_{\alpha}\right|^{2}}{w_{\gamma_{1} \ldots \gamma_{k}, \gamma_{1} \ldots \gamma_{k}}} \\
& -\sum_{\left(\gamma_{1}, \ldots, \gamma_{k}\right) \in G} \sum_{\nu \leq \beta_{1}<\cdots<\beta_{k-m} \leq n-m} \frac{\left|\left(w_{\beta_{1} \ldots \beta_{k-m}(n-m+1) \ldots n, \gamma_{1} \ldots \gamma_{k}}\right) \bar{\alpha}\right|^{2}}{w_{\gamma_{1} \ldots \gamma_{k}, \gamma_{1} \ldots \gamma_{k}}} .
\end{aligned}
$$

Observe that

$$
\begin{aligned}
& \sum_{\nu \leq \beta_{1}<\cdots<\beta_{k-m} \leq n-m}\left(w_{\beta_{1} \ldots \beta_{k-m}(n-m+1) \ldots n, \beta_{1} \ldots \beta_{k-m}(n-m+1) \ldots n}\right)_{\alpha \bar{\alpha}} \\
= & \sum_{\nu \leq \beta_{1}<\cdots<\beta_{k-m} \leq n-m}\left(\sum_{i=1}^{k-m} u_{\beta_{i} \bar{\beta}_{i} \alpha \bar{\alpha}}+\sum_{\gamma=n-m+1}^{n} u_{\gamma \bar{\gamma} \alpha \bar{\alpha}}\right) \\
= & \left(\begin{array}{c}
n-m-\nu \\
k-m-1
\end{array}\right) \sum_{\beta=\nu}^{n-m} u_{\beta \bar{\beta} \alpha \bar{\alpha}}+\left(\begin{array}{c}
n-m-\nu+1 \\
k-m
\end{array}\right) \sum_{\gamma=n-m+1}^{n} u_{\gamma \bar{\gamma} \alpha \bar{\alpha}} .
\end{aligned}
$$

Next, (4.15) plus (4.16) is equal to

$$
\begin{aligned}
& -\sum_{\gamma=1}^{\nu-1} \sum_{\left(\beta_{1}, \ldots, \beta_{k-m}\right)} \sum_{j=1}^{k-m} \frac{\left|u_{\beta_{j} \bar{\gamma} \alpha}\right|^{2}+\left|u_{\beta_{j} \bar{\gamma} \bar{\alpha}}\right|^{2}}{u_{\gamma \bar{\gamma}}+\sum_{1 \leq l \leq k-m, l \neq j} u_{\beta_{l} \bar{\beta}_{l}}+\sum_{\tau=n-m+1}^{n} u_{\tau \bar{\tau}}} \\
& -\sum_{\gamma=1}^{\nu-1} \sum_{\left(\beta_{1}, \ldots, \beta_{k-m}\right)} \sum_{j=1}^{m} \frac{\left|u_{(n-m+j) \bar{\gamma} \alpha}\right|^{2}+\left|u_{(n-m+j) \bar{\gamma} \bar{\alpha}}\right|^{2}}{u_{\gamma \bar{\gamma}}+\sum_{i=1}^{k-m} u_{\beta_{i} \bar{\beta}_{i}}+\sum_{1 \leq l \leq m, l \neq j} u_{(n-m+l)} \overline{(n-m+l)}} \\
& -\sum_{\gamma=\nu}^{n-m} \sum_{\left(\beta_{1}, \ldots, \beta_{k-m}\right)}^{\circ} \sum_{j=1}^{m} \frac{\left|u_{(n-m+j) \bar{\gamma} \alpha}\right|^{2}+\left|u_{(n-m+j) \bar{\gamma} \bar{\alpha}}\right|^{2}}{u_{\gamma \bar{\gamma}}+\sum_{i=1}^{k-m} u_{\beta_{i} \bar{\beta}_{i}}+\sum_{1 \leq l \leq m, l \neq j} u_{(n-m+l)} \overline{(n-m+l)}}
\end{aligned}
$$

in which $\sum_{\left(\beta_{1}, \ldots, \beta_{k-m}\right)}$ denotes the summation over all $\nu \leq \beta_{1}<\cdots<\beta_{k-m} \leq n-m$, while $\sum_{\left(\beta_{1}, \ldots, \beta_{k-m}\right)}^{\circ}$ denotes the summation over all $\nu \leq \beta_{1}<\cdots<\beta_{k-m} \leq n-m$ and $\beta_{j} \neq \gamma$ for all $j=1, \ldots, k-m$. By (4.7) we can simplify the above expression as 
follows:

$$
\begin{aligned}
& -\left(\begin{array}{c}
n-m-\nu \\
k-m-1
\end{array}\right) \sum_{\gamma=1}^{\nu-1} \sum_{\beta=\nu}^{n-m} \frac{\left|u_{\beta \bar{\gamma} \alpha}\right|^{2}+\left|u_{\beta \bar{\gamma} \bar{\alpha}}\right|^{2}}{u_{\gamma \bar{\gamma}}-u_{\nu \bar{\nu}}} \\
& -\left(\begin{array}{c}
n-m-\nu-1 \\
k-m
\end{array}\right) \sum_{\gamma=1}^{\nu-1} \sum_{\eta=n-m+1}^{n} \frac{\left|u_{\eta \bar{\gamma} \alpha}\right|^{2}+\left|u_{\eta \bar{\gamma} \bar{\alpha}}\right|^{2}}{u_{\gamma \bar{\gamma}}-u_{\eta \bar{\eta}}} \\
& -\left(\begin{array}{c}
n-m-\nu \\
k-m
\end{array}\right) \sum_{\beta=\nu}^{n-m} \sum_{\eta=n-m+1}^{n} \frac{\left|u_{\eta \bar{\beta} \alpha}\right|^{2}+\left|u_{\eta \bar{\beta} \bar{\alpha}}\right|^{2}}{u_{\beta \bar{\beta}}-u_{\eta \bar{\eta}}} .
\end{aligned}
$$

Thus,

$$
\begin{aligned}
& Q^{-1} \Delta \phi=Q^{-1} \sum_{\alpha=1}^{n} \phi_{\alpha \bar{\alpha}} \\
& \lesssim\left(\begin{array}{c}
n-m-\nu \\
k-m-1
\end{array}\right) \sum_{\beta=\nu}^{n-m} f_{\beta \bar{\beta}}+\left(\begin{array}{c}
n-m-\nu+1 \\
k-m
\end{array}\right) \sum_{\gamma=n-m+1}^{n} f_{\gamma \bar{\gamma}} \\
& -\left(\begin{array}{l}
n-m-\nu \\
k-m-1
\end{array}\right) \sum_{\alpha=1}^{n} \sum_{\gamma=1}^{\nu-1} \sum_{\beta=\nu}^{n-m} \frac{\left|u_{\beta \bar{\gamma} \alpha}\right|^{2}+\left|u_{\beta \bar{\gamma} \bar{\alpha}}\right|^{2}}{u_{\gamma \bar{\gamma}}-u_{\nu \bar{\nu}}} \\
& -\left(\begin{array}{c}
n-m-\nu-1 \\
k-m
\end{array}\right) \sum_{\alpha=1}^{n} \sum_{\gamma=1}^{\nu-1} \sum_{\eta=n-m+1}^{n} \frac{\left|u_{\eta \bar{\gamma} \alpha}\right|^{2}+\left|u_{\eta \bar{\gamma} \alpha}\right|^{2}}{u_{\gamma \bar{\gamma}}-u_{\eta \bar{\eta}}} \\
& -\left(\begin{array}{c}
n-m-\nu \\
k-m
\end{array}\right) \sum_{\alpha=1}^{n} \sum_{\beta=\nu}^{n-m} \sum_{\eta=n-m+1}^{n} \frac{\left|u_{\eta \bar{\beta} \alpha}\right|^{2}+\left|u_{\eta \bar{\beta} \bar{\alpha}}\right|^{2}}{u_{\beta \bar{\beta}}-u_{\eta \bar{\eta}}} \\
& \lesssim\left[\left(\begin{array}{l}
n-m-\nu \\
k-m-1
\end{array}\right) \sum_{\beta=\nu}^{n-m} f_{\beta \bar{\beta}}-\left(\begin{array}{l}
n-m-\nu \\
k-m-1
\end{array}\right) \sum_{\beta=\nu}^{n-m} \sum_{\gamma=1}^{\nu-1} \frac{\left|u_{\gamma \bar{\gamma} \beta}\right|^{2}}{u_{\gamma \bar{\gamma}}-u_{\nu \bar{\nu}}}\right. \\
& \left.-\left(\begin{array}{c}
n-m-\nu \\
k-m
\end{array}\right) \sum_{\beta=\nu}^{n-m} \sum_{\eta=n-m+1}^{n} \frac{\left|u_{\eta \bar{\eta} \beta}\right|^{2}}{u_{\beta \bar{\beta}}-u_{\eta \bar{\eta}}}\right] \\
& +\left[\left(\begin{array}{c}
n-m-\nu+1 \\
k-m
\end{array}\right) \sum_{\eta=n-m+1}^{n} f_{\eta \bar{\eta}}-\left(\begin{array}{c}
n-m-\nu \\
k-m
\end{array}\right) \sum_{\eta=n-m+1}^{n} \sum_{\beta=\nu}^{n-m} \frac{\left|u_{\beta \bar{\beta}}\right|^{2}}{u_{\beta \bar{\beta}}-u_{\eta \bar{\eta}}}\right. \\
& \left.-\left(\begin{array}{c}
n-m-\nu+1 \\
k-m
\end{array}\right) \sum_{\eta=n-m+1}^{n} \sum_{\gamma=1}^{\nu-1} \frac{\left|u_{\gamma \bar{\gamma} \eta}\right|^{2}}{u_{\gamma \bar{\gamma}}-u_{\eta \bar{\eta}}}\right] .
\end{aligned}
$$


It then follows from Proposition 4.8 and Proposition 4.9 below that

$$
\begin{aligned}
Q^{-1} \Delta \phi & \lesssim\left(\begin{array}{c}
n-m-\nu \\
k-m-1
\end{array}\right) \sum_{\beta=\nu}^{n-m}\left(f_{\beta \bar{\beta}}-\frac{\left|f_{\beta}\right|^{2}}{k f}\right) \\
& +\left(\begin{array}{c}
n-m-\nu+1 \\
k-m
\end{array}\right) \sum_{\eta=n-m+1}^{n}\left(f_{\eta \bar{\eta}}-\frac{\left|f_{\eta}\right|^{2}}{k f}\right) \\
& =\sum_{\nu \leq \beta_{1}<\cdots<\beta_{k-m} \leq n-m}\left[\sum_{i=1}^{k-m}\left(f_{\beta_{i} \bar{\beta}_{i}}-\frac{\left|f_{\beta_{i}}\right|^{2}}{k f}\right)+\sum_{\eta=n-m+1}^{n}\left(f_{\eta \bar{\eta}}-\frac{\left|f_{\eta}\right|^{2}}{k f}\right)\right]
\end{aligned}
$$

Therefore, $\Delta \phi \lesssim 0$ if the Hermitian matrix $\left(k f f_{\alpha \bar{\beta}}-f_{\alpha} f_{\bar{\beta}}\right)$ is $k$-concave in $\Omega$. $\square$

Proposition 4.8. For each $\zeta=\nu, \nu+1, \ldots, n-m$, we have

$$
\begin{aligned}
\left(\begin{array}{c}
n-m-\nu \\
k-m-1
\end{array}\right) & \sum_{\gamma=1}^{\nu-1} \frac{\left|u_{\gamma \bar{\gamma} \zeta}\right|^{2}}{u_{\gamma \bar{\gamma}}-u_{\nu \bar{\nu}}}+\left(\begin{array}{c}
n-m-\nu \\
k-m
\end{array}\right) \sum_{\eta=n-m+1}^{n} \frac{\left|u_{\eta \bar{\eta} \zeta}\right|^{2}}{u_{\zeta \bar{\zeta}}-u_{\eta \bar{\eta}}} \\
& \gtrsim\left(\begin{array}{l}
n-m-\nu \\
k-m-1
\end{array}\right) \frac{(k-1)\left|f_{\zeta}\right|^{2}}{k f} .
\end{aligned}
$$

Proof. Let

$$
A=\left(\begin{array}{c}
n-m-\nu \\
k-m-1
\end{array}\right), \quad C=\left(\begin{array}{c}
n-m-\nu \\
k-m
\end{array}\right)
$$

By the Schwarz inequality,

$$
\begin{aligned}
& A \sum_{\gamma=1}^{\nu-1} \frac{\left|u_{\gamma \bar{\gamma} \zeta}\right|^{2}}{u_{\gamma \bar{\gamma}}-u_{\nu \bar{\nu}}}+C \sum_{\eta=n-m+1}^{n} \frac{\left|u_{\eta \bar{\eta} \zeta}\right|^{2}}{u_{\zeta \bar{\zeta}}-u_{\eta \bar{\eta}}} \\
& \gtrsim \frac{\left|A \sum_{\gamma=1}^{\nu-1} u_{\gamma \bar{\gamma} \zeta}-C \sum_{\eta=n-m+1}^{n} u_{\eta \bar{\eta} \zeta}\right|^{2}}{A \sum_{\gamma=1}^{\nu-1}\left(u_{\gamma \bar{\gamma}}-u_{\nu \bar{\nu}}\right)+C \sum_{\eta=n-m+1}^{n}\left(u_{\nu \bar{\nu}}-u_{\eta \bar{\eta}}\right)} \\
& \gtrsim A^{2}\left|f_{\eta}\right|^{2}\left(\frac{A k}{k-m} \sum_{\gamma=1}^{\nu-1} u_{\gamma \bar{\gamma}}+C k u_{\nu \bar{\nu}}\right)^{-1} \\
& \sim \frac{A(k-m)}{k}\left|f_{\eta}\right|^{2} f^{-1} .
\end{aligned}
$$

In (4.17) we have used (4.14) and that

$$
-(\nu-1) u_{\nu \bar{\nu}} \sim \frac{\nu-1}{k-m} \sum_{\eta=n-m+1}^{n} u_{\eta \bar{\eta}} \lesssim \frac{1}{k-m} \sum_{\gamma=1}^{\nu-1} u_{\gamma \bar{\gamma}},
$$

while (4.18) follows from (4.11) and

$$
C=\frac{n-k-\nu+1}{k-m} A
$$

This completes the proof. $\square$ 
Proposition 4.9. For each $\eta=n-m+1, \ldots, n$, we have

$$
\begin{aligned}
\left(\begin{array}{c}
n-m-\nu+1 \\
k-m
\end{array}\right) & \sum_{\gamma=1}^{\nu-1} \frac{\left|u_{\gamma \bar{\gamma} \eta}\right|^{2}}{u_{\gamma \bar{\gamma}}-u_{\eta \bar{\eta}}}+\left(\begin{array}{c}
n-m-\nu \\
k-m
\end{array}\right) \sum_{\beta=\nu}^{n-m} \frac{\left|u_{\beta \bar{\beta} \eta}\right|^{2}}{u_{\beta \bar{\beta}}-u_{\eta \bar{\eta}}} \\
& \gtrsim\left(\begin{array}{c}
n-m-\nu+1 \\
k-m
\end{array}\right) \frac{\left|f_{\eta}\right|^{2}}{k f} .
\end{aligned}
$$

Proof. For convenience, let us denote by

$$
B=\left(\begin{array}{c}
n-m-\nu+1 \\
k-m
\end{array}\right), \quad C=\left(\begin{array}{c}
n-m-\nu \\
k-m
\end{array}\right) .
$$

First, notice the fact that

$$
-u_{\eta \bar{\eta}} \lesssim(k-1) u_{\nu \bar{\nu}}
$$

Indeed,

$$
\begin{aligned}
0 & \leq w_{(n-k+1) \ldots n,(n-k+1) \ldots n}=\sum_{\gamma=n-k+1}^{n-m} u_{\gamma \bar{\gamma}}+\sum_{\zeta=n-m+1}^{n} u_{\zeta \bar{\zeta}} \\
& \sim(k-m) u_{\nu \bar{\nu}}+\sum_{\zeta=n-m+1}^{n} u_{\zeta \bar{\zeta}}(\text { by }(4.7)) \\
& \lesssim(k-1) u_{\nu \bar{\nu}}+u_{\eta \bar{\eta}}, \quad(\text { by }(4.1)) .
\end{aligned}
$$

Now we apply the Schwarz inequality to obtain that

$$
\begin{aligned}
& B \sum_{\gamma=1}^{\nu-1} \frac{\left|u_{\gamma \bar{\gamma} \eta}\right|^{2}}{u_{\gamma \bar{\gamma}}-u_{\eta \bar{\eta}}}+C \sum_{\beta=\nu}^{n-m} \frac{\left|u_{\beta \bar{\beta}}\right|^{2}}{u_{\beta \bar{\beta}}-u_{\eta \bar{\eta}}} \\
& \geq\left|B \sum_{\gamma=1}^{\nu-1} u_{\gamma \bar{\gamma} \eta}+C \sum_{\beta=\nu}^{n-m} u_{\beta \bar{\beta} \eta}\right|^{2}\left(B \sum_{\gamma=1}^{\nu-1} u_{\gamma \bar{\gamma}}+C \sum_{\beta=\nu}^{n-m} u_{\beta \bar{\beta}}-B(n-k) u_{\eta \bar{\eta}}\right)^{-1} \\
& \sim B\left|f_{\eta}\right|^{2}\left(f-(n-k) u_{\eta \bar{\eta}}\right)^{-1} \quad(\text { by (4.13)) } \\
& \gtrsim B\left|f_{\eta}\right|^{2}\left(f+(n-k)(k-1) u_{\nu \bar{\nu}}\right)^{-1} \quad(\text { by }(4.19)) \\
& \gtrsim B\left|f_{\eta}\right|^{2}(k f)^{-1}, \quad(\text { by }(4.12)) .
\end{aligned}
$$

Therefore, the proof of Theorem 1.1 is completed.

\section{REFERENCES}

[1] O. Alvarez, J. M. Lasry and P. L. Lions, Convexity viscosity solutions and state constraints, J. Math. Pures Appl., 76 (1997), pp. 265-288.

[2] B. J. BIAN AND P. GUAN, A microscopic convexity principle for nonlinear partial differential equations, to appear in Invent. Math. (Online First DOI: 10.1007/s00222-009-0179-5). 
[3] C. Böhm And B. Wilking, Manifolds with positive curvature operators are space forms, Ann. of Math. (2), 167:3 (2008), pp. 1079-1097.

[4] L. Caffarelli and A. Friedman, Convexity of solutions of some semilinear elliptic equations, Duke Math. J., 52 (1985), pp. 431-455.

[5] L. Caffarelli, P. Guan AND X. N. MA, A constant rank theorem for solutions of fully nonlinear elliptic equations, Comm. Pure and Appl. Math., 60:12 (2007), pp. 1769-1791.

[6] L. Caffarelli, L. Nirenberg And J. Spruck, The Dirichlet problem for nonlinear second order elliptic equations, III: Functions of the eigenvalues of the Hessian, Acta Math., 155 (1985), pp. 261-301.

[7] H. Chen, Pointwise 1/4-pinched 4-manifolds, Ann. Global Anal. Geom., 9 (1991), pp. 161-176.

[8] E. M. Chirka, Introduction to the geometry of CR-manifolds, Russian Math. Surveys, 46:1 (1991), pp. 95-97.

[9] A. Fraser, Minimal disks and two-convex hypersurfaces, Amer. J. Math., 124:3 (2002), pp. $483-493$.

[10] A. Fraser, Index estimates for minimal surfaces and $k$-convexity, Proc. Amer. Math. Soc., 135:11 (2007), pp. 3733-3744.

[11] P. Guan, C. S. Lin and X. N. MA, The Christoffel-Minkowski problem II: Weingarten curvature equations, Chinese Ann. of Math. Series B, 27:6 (2006), pp. 595-614.

[12] P. Guan AND X. N. Ma, The Christoffel-Minkowski problem I: convexity of solutions of a Hessian equations, Invent. Math., 151 (2003), pp. 553-577.

[13] F. HAN, X. N. MA AND D. Wu, An existence theorem of $k$-convex hypersurface with prescribed mean curvature, preprint, 2008.

[14] F. R. Harvey and H. B. Lawson, Dirichlet duality and the nonlinear Dirichlet problem, Comm. Pure and Appl. Math., Published Online: Sep 25 2008,DOI:10.1002/cpa.20265.

[15] G. Huisken and C. Sinestrari, Mean curvature flow with surgeries of two-convex hypersurfaces, Inventiones Math., DOI 10.1007/s00222-008-0148-4.

[16] B. KAWOHL, A remark on N.Korevaar's concavity maximum principle and on the asymptotic uniqueness of solutions to the plasma problem, Math. Methods Appl. Sci., 8 (1986), pp. 93101.

[17] A. U. Kennington, Power concavity and boundary value problems, Indiana Univ. Math. J., 34 (1985), pp. 687-704.

[18] S. KoBAYASHI AND H. Wu, On holomorphic sections of certain Hermitian vector bundles, Math. Ann., 189 (1970), pp. 1-4.

[19] N. J. KorevaAr, Capillary surface convexity above convex domains, Indiana Univ. Math. J., 32 (1983), pp. 73-81.

[20] N. J. KorevaAR, Convex solutions to nonlinear elliptic and parabolic boundary value problems, Indiana Univ. Math. J., 32 (1983), pp. 603-614.

[21] N. J. KorevaAr, Convexity properties of solutions to elliptic P. D. E.'s, Variational Methods for free surface interfaces, Springer, 1986, pp. 115-121.

[22] N. J. KorevaAR AND J. LewIS, Convex solutions of certain elliptic equations have constant rank hessians, Arch. Rational Mech. Anal., 91 (1987), pp. 19-32.

[23] Q. LI, Constant rank theorem in complex variables, to appear in Indiana Univ. Math. J., 2008.

[24] X. Ma And G. Marinescu, Holomorphic Morse Inequalities and Bergman Kernels, Progress in Mathematics, vol. 254, Birkhäuser, 2007.

[25] J. D. Moore And T. Schulte, Minimal disks and compact hypersurfaces in Euclidean space, Proc. Amer. Math. Soc., 94:2 (1985), pp. 321-328.

[26] J. P. SHA, Handlebodies and p-convexity, J. Differential Geom., 25:3 (1987), pp. 353-361.

[27] J. P. ShA, p-convex Riemannian manifolds, Invent. Math., 83:3 (1986), pp. 437-447

[28] I. Singer, B. Wong, S. T. Yau and Stephen S. T. Yau, An estimate of gap of the first two eigenvalues in the Schrodinger operator, Ann. Scuola Norm. Sup. Pisa Cl. Sci.(4), 12 (1985), pp. 319-333.

[29] M. P. Tsui And M. T.Wang, Mean curvature flows and isotopy of maps between spheres, Comm. Pure Appl. Math., 57:8 (2004), pp. 1110-1126.

[30] H. Wu, Manifolds of partially positive curvature, Indiana Univ. Math. J., 36:3 (1987), pp. 525548. 
F. HAN, X.-N. MA AND D. WU 